FU-PCG-15

\title{
Current density and conductivity through modified gravity in the graphene with defects
}

\author{
Alireza Sepehri ${ }^{a, b}$ Richard Pincak $^{c, d}$ Kazuharu Bamba $^{e}$ Salvatore Capozziello ${ }^{f, g, h, i}$ \\ Emmanuel N. Saridakis ${ }^{j, k, l}$ \\ ${ }^{a}$ Faculty of Physics, Shahid Bahonar University, P.O. Box 76175, Kerman, Iran \\ ${ }^{b}$ Research Institute for Astronomy and Astrophysics of Maragha (RIAAM), P.O. Box 55134-441, \\ Maragha, Iran \\ ${ }^{c}$ Institute of Experimental Physics, Slovak Academy of Sciences, Watsonova 47,043 53 Kosice, \\ Slovak Republic \\ ${ }^{d}$ Bogoliubov Laboratory of Theoretical Physics, Joint Institute for Nuclear Research, 141980 Dubna, \\ Moscow region, Russia \\ ${ }^{e}$ Division of Human Support System, Faculty of Symbiotic Systems Science, Fukushima University, \\ Fukushima 960-1296, Japan \\ ${ }^{f}$ Dipartimento di Fisica, Universita di Napoli Federico II, I-80126 - Napoli, Italy \\ ${ }^{g}$ INFN Sez. di Napoli, Compl. Univ. di Monte S. Angelo, Edificio G, I-80126 - Napoli, Italy \\ ${ }^{h}$ Gran Sasso Science Institute (INFN), Viale F. Crispi, 7, I-67100 L'Aquila, Italy \\ ${ }^{j}$ Instituto de Física, Pontificia Universidad de Católica de Valparaíso, Casilla 4950, Valparaíso, \\ Chile \\ ${ }^{k}$ CASPER, Physics Department, Baylor University, Waco, TX 76798-7310, USA \\ ${ }^{l}$ Physics Division, National Technical University of Athens, 15780 Zografou Campus, Athens, \\ Greece \\ E-mail: alireza.sepehri@uk.ac.ir, pincak@saske.sk, \\ bamba@sss.fukushima-u.ac.jp, capozziello@na.infn.it, \\ Emmanuel_Saridakis@baylor.edu
}

ABSTRACT: We propose a model describing the evolution of the free electron current density in graphene. Based on the concept of Mp-branes, we perform the analysis using the difference between curvatures of parallel and antiparallel spins. In such a framework an effective graviton emerges in the form of gauge field exchange between electrons. In a plain graphene system, the curvatures produced by both kinds of spins neutralize each other. However, in the presence of defects, the inequality between curvatures leads to the emergence of current density, modified gravity and conductivity. Depending on the type of the defects, the resulting current density can be negative or positive.

KeYworDS: Graphene; Defects; M-theory; Modified gravity. 


\section{Contents}

1 Introduction 1

2 The current density in graphene $\quad 4$

3 The current density in graphene in presence of various defects $\quad 8$

$\begin{array}{llr}3.1 & \text { Graphene without defects (hexagonal) } & 9\end{array}$

$\begin{array}{ll}3.2 & \text { Graphene with heptagonal defects } \\ & 11\end{array}$

$\begin{array}{ll}3.3 & \text { Graphene with pentagonal defects } \\ \end{array}$

4 The current density in a graphene wormhole $\quad 14$

$\begin{array}{llr}5 & \text { Summary and Discussion } & 16\end{array}$

A The action components (2.3) in terms of couplings of parallel and anti$\begin{array}{ll}\text { parallel spins } & 19\end{array}$

B Expression of $\left\langle\partial^{b} \partial^{a} X^{i}, \partial_{b} \partial_{a} X^{i}\right\rangle$ in terms of curvatures $\quad 21$

$\begin{array}{ll}\text { C Defects and } f(R) \text { gravity } & 21\end{array}$

\section{Introduction}

During the last years, graphene has been in the focus of frontier research, since its mass energy excitation for fermions is low, as it emerges from the symmetries of the honeycomb lattice $[1-7,9]$. This research has led to many important results. Amongst others, firstly, the surface free energy and the elastic constants (i.e. the Lame parameters such as Poisson ratio and Youngs modulus,) of graphene flakes, have been obtained on the level of the density functional theory. In particular, it has been shown that the Lame parameters in small graphene flakes may differ from the bulk values by thirty percent, for hydrogenated zig-zag edges which originate from the edge of the flake that compresses the interior [1]. Secondly, other investigations show that graphene with (effective) vacancy disorder is a physical representative of dirty d-wave superconductors [2]. Thirdly, the density of states (DoS) of this system has been obtained numerically and within the self-consistent T-matrix approximation (SCTMA) in the presence of vacancies [3-5]. Fourthly, other works have considered quantum size effects in armchair graphene nanoribbons (AGNRs) with hydrogen termination via density functional theory (DFT) in Kohn-Sham formulation, and they have obtained the electronic structure of this system and predicted a threefold periodicity of the excitation gap with ribbon width [6]. Furthermore, in another scenario the local current density in pristine armchair graphene nanoribbons with varying width has been calculated 
and it was found that the response of the current to functionalizing adsorbates is very sensitive to their placement: adsorbates located within the current filaments lead to strong backscattering, while adsorbates placed in other regions have almost no impact at all [7]. Moreover, in [8] it was shown that geometry has crucial effects on the electronic properties of the graphene. Also, the spin-dependent zero-bias conductance, in armchair graphene nanoribbons with hydrogen adsorbates, has been derived and the spin-orbit interaction has been considered. It has been observed that the spin-flip conductance can reach the same order of magnitude as the spin-conserving one, due to exchange-mediated spin scattering [9]. Additionally, in [10] the authors studied the electrically charged black holes in Palatini formalism of $f(R)$ gravity, and they have argued that a form $f(R)=R \pm \alpha R^{2} / R_{P}$ (with $R_{P}$ the Planck curvature) could induce different geometrical structures in terms of inner black holes horizons. In a different scenario it was shown that Palatini $f(R)$ gravity may lead to a geometry in which point-like space-time singularity in four dimensional classical models is replaced by a finite size wormhole structure, and this non-singular spacetime appears despite the existence of curvature divergences at the wormhole throat [11]. Finally, in [12] the authors explained that microscopic wormholes which are created in modified gravity theories like $f(R)$ gravity have a role analogous to that of defects in crystals, which indicates the possible connection of solid-state and modified-gravity effects.

According to some considerations, massless two-dimensional Dirac fermions in a graphene behave in a way that can be described in terms of both quantum field theory and condensed matter physics. The formulated connection considers the role of gauge fields [13], and motivated by this research we propose the model of the electronic transport in graphene in which Mp-branes [14-19], known from cosmology, appear. In the related superstring theory, initially there were only open scalar strings, which were later attached to zero dimensional objects, called M0-branes. This attachment is similar to the attachment of strings to a point-like one zero dimensional page. In fact, in string theory, each open string is attached from each end to one point or zero dimensional page which called M0. Moreover, at the beginning the shapes of all strings in respect to each other and to M0-branes are the same, they have no spin, and hence we cannot observe any difference between them. That is why we only have scalar strings at this stage. However, through interactions between strings and M0-branes, the shapes of strings in respect to each other are changed and they become different. Some of them gain properties of gauge fields with spin one, some obtain properties of fields with spin 2, while some other achieve properties of other spins. Nevertheless, at the beginning, no gauge fields and fermions were present [15]. Then, the M0-branes were joined and constructed a system of M1 and anti-M1-branes connected by a wormhole, a system named BIon [16-19]. As a next step, the M0-branes glued to each other symmetrically, with the upper and lower parts of M1-brane being the same, and hence gauge fields were produced. Moreover, by linking M0-branes anti-symmetrically, fermions were created [15, 18]. Finally, these M1-BIons were connected and formed M3-BIons, which were a configuration of an M3, an anti-M3-brane connected by a wormhole [16-18]. In this context/picture our Universe would be located on one of these M3-branes and its evolution is determined by interaction of branes in extra dimensions [14-19].

Furthermore, there are works in the literature in which dark matter and cosmologi- 
cal acceleration are produced by braneworld scenarios. In particular, in [20] the authors showed that in the context of brane-world models with low tension $\tau=f^{4}$, massive brane fluctuations are natural dark matter candidates, while the present abundances for both hot(warm) and cold branons were obtained in terms of the branon mass $M$ and the tension scale $f$. These results were in agreement with the recent experimental bounds on these parameters. Additionally, in a different braneworld model the main phenomenology associated with disformal scalars coupled to the Standard Model fields were considered, and it was argued that these fields can serve as natural dark matter candidates since they are massive and weakly coupled [21]. Finally, in [22] the possible detection of branon dark matter in experimental data was discussed. For reviews on braneworld models which produce cosmological acceleration see [23-25].

A similar model can be constructed for condensed matter systems. In this paper, we propose an approach leading to modified gravity [26, 27] due to defects in graphene, and we calculate the current density of free electrons in terms of the curvature of the system. Each atom in graphene has three bound electrons, which are paired with the electrons of other atoms, and one free electron whose motion leads to the emergence of conductivity. Without free electrons, there is a high symmetry in this system, and graphene behaves similarly to M0-brane, where paired electrons behave like scalar fields. However, by the motion of free electrons, the system symmetry is broken and two gauge fields are created. These gauge fields play the role of gravitons and produce the gravity between anti-parallel spins and anti-gravity between parallel spins. For a graphene without defects, the curvature produced by non-identical spins is neutralized by the curvature of identical fermions, and the total curvature of the system becomes zero. This curvature $(R)$ is related to the energymomentum tensor $\left(T_{\mu}^{\nu}=\operatorname{diag}[-p,-p,-p, \rho]\right)$ by the relation $\left(T_{\mu \nu}=R_{\mu \nu}-\frac{1}{2} R g_{\mu n u}\right)$ and thus the imposed momentum to the electron is zero. Along this context, the meaning of anti-gravity becomes more transparent. In particular, when the curvature is zero the energy momentum tensor is zero $\left(R \approx T_{\mu}^{\nu}=0\right)$ and consequently its components such as energy density and momentums are zero. Since force is related to momentum changes we deduce that the applied force to particles is zero. This implies that free electrons do not move in a certain path, and their current density becomes zero and thus conductivity disappears. On the other hand, the existence of suitable types of defects leads anti-parallel spins to come closer mutually, and therefore their curvature increases. In this context, modified gravity emerges and applies momentum to free electrons. Consequently, free electrons move in a special path, the current density increases and conductivity grows. Similarly, other types of defects make parallel spins to approach each other, thus increasing the curvature leading to the emergence of (modified) anti-gravity. Contrary to the fact that gravity produces attractive force, anti-gravity creates a repulsive force between bodies, objects and particles. In anti-gravity case, momentum is applied to free electrons in opposite directions, and hence the sign of current density reverses. When the curvature is negative, it's related effective energy-momentum tensor is negative and consequently, the applied force becomes negative. Consequently, particles move mutually away.

The outline of the paper is the following. In Section 2 we obtain the current density in terms of inequality between curvatures of parallel and anti-parallel spins. In Section 3 we 
show that this density is zero for standard graphene, positive for pentagonal defects and negative for heptagonal defects. In Section 4 we obtain the current density in graphene wormholes. Finally, Section 5 is devoted to discussion and conclusions.

\section{The current density in graphene}

Let us introduce the concepts of Mp-branes in order to extract the current density of free electrons in terms of inequality between curvatures of parallel spins and anti-parallel spins. In order to achieve this result, we apply the method used in $[14,15,18]$ for calculating the energy in terms of curvature, and we write the explicit form of curvatures in terms of couplings of parallel and anti-parallel spins.

Let us begin by considering the scalar fields $X^{M}=X_{\alpha}^{M} T^{\alpha}$, where the generators $T^{\alpha}$ of the Lie 3-algebra satisfy the 3-dimensional Nambu-Poisson brackets [28-34]

$$
\begin{aligned}
& {\left[T^{\alpha}, T^{\beta}, T^{\gamma}\right]=f_{\eta}^{\alpha \beta \gamma} T^{\eta}} \\
& {\left[X^{M}, X^{N}, X^{L}\right]=\left[X_{\alpha}^{M} T^{\alpha}, X_{\beta}^{N} T^{\beta}, X_{\gamma}^{L} T^{\gamma}\right],}
\end{aligned}
$$

with $f_{\eta}^{\alpha \beta \gamma}$ the structure constants. Let us also consider a 2-form gauge field $A_{a b}$. In this case, the full action in M-theory reads [14, 15, 18]:

$$
S_{\text {co-Graphene }}=\int d^{3} x \sum_{n=1}^{U} \beta_{n}\left(\delta_{b_{1} b_{2} \ldots b_{n}}^{a_{1}, a_{2} \ldots a_{n}} L_{a_{1}}^{b_{1}} \ldots L_{a_{n}}^{b_{n}}\right)^{1 / 2},
$$

where

$$
(L)_{b}^{a}=\delta_{a}^{b} S \operatorname{Tr}\left\{-\operatorname{det}\left(P_{a b c}\left[E_{m n l}+E_{m i j}\left(Q^{-1}-\delta\right)^{i j k} E_{k l n}\right]+\lambda F_{a b c}\right) \operatorname{det}\left(Q_{j, k}^{i}\right)\right\},
$$

with

$$
\begin{aligned}
E_{m n l}^{\alpha, \beta, \gamma} & =G_{m n l}^{\alpha, \beta, \gamma}+B_{m n l}^{\alpha, \beta, \gamma}, \\
Q_{j, k}^{i} & =\delta_{j, k}^{i}+i \lambda\left[X_{\alpha}^{j} T^{\alpha}, X_{\beta}^{k} T^{\beta}, X_{\gamma}^{k^{\prime}} T^{\gamma}\right] E_{k^{\prime} j l}^{\alpha, \beta, \gamma}, \\
F_{a b c} & =\partial_{a} A_{b c}-\partial_{b} A_{c a}+\partial_{c} A_{a b} .
\end{aligned}
$$

In the above expressions $G_{m n l}=g_{m n} \delta_{n, l}^{n^{\prime}}+\partial_{m} X^{i} \partial_{n^{\prime}} X^{i} \sum_{j}\left(X^{j}\right)^{2} \delta_{n, l}^{n^{\prime}}+\frac{1}{2}\left\langle\partial^{b} \partial^{a} X^{i}, \partial_{b} \partial_{a} X^{i}\right\rangle$, $P_{a b c}$ is the pull-back of scalars, and $S T r$ stands for the symmetric trace of products. In graphene, the scalar fields denote the pairs which are produced by pairing anti-parallel electrons. Similarly, $\lambda=2 \pi l_{s}^{2}$ where, in M-theory, $l_{s}$ is the string length, which, in graphene, is just the separation distance between atoms. Additionally, $\beta_{n}$ are constants, related to different atoms in graphene. Concerning the index notation, note that, in graphene, $a, b$ denote indices of pairs on each atom, while $i, j$ refer to indices of free pairs corresponding to free electrons. Finally, $U$ denotes the number of atoms and $p$ is the number of pairs in each atom.

Applying action (2.2) to graphene, we assume that the scalar fields $X^{i}$ play the role of pairs of anti-parallel electrons and the 2-form gauge tensor fields $A_{a b}$ play the role of gravitons which are exchanged between electrons. This assumption is justified since, in 
graphene, the three electrons of each atom are paired with three electrons of another atom by exchanging 2-form gauge fields forming spinless pairs which can be treated as scalars. Moreover, free electrons are represented by $\psi$, while electrons in each pair are denoted by $\Psi$. Hence, with these positions, we can define [18]:

$$
\begin{aligned}
& A_{a b} \rightarrow \psi_{a}^{U} \psi_{b}^{L}-\psi_{a}^{L} \psi_{b}^{U} \\
& X \rightarrow \psi_{a}^{U} A^{a b} \psi_{b}^{L}-\psi_{a}^{L} A^{a b} \psi_{b}^{U}+\Psi_{a}^{U} A^{a b} \Psi_{b}^{L}-\Psi_{a}^{L} A^{a b} \Psi_{b}^{U}+\Psi_{a}^{U} A^{a b} \psi_{b}^{L}-\psi_{a}^{L} A^{a b} \Psi_{b}^{U} \\
& \partial_{a}=\partial_{a}^{U}+\partial_{a}^{L} \\
& \partial_{a}^{U} \psi_{a}^{U}=1, \partial_{a}^{L} \psi_{a}^{L}=1 .
\end{aligned}
$$

Using these definitions we can make the splitting [18]:

$$
\left\langle F^{a b c}, F_{a b c}\right\rangle_{t o t} \equiv\left\langle F^{a b c}, F_{a b c}\right\rangle_{F r e e-F r e e}+\left\langle F^{a b c}, F_{a b c}\right\rangle_{F r e e-B o u n d}+\left\langle F^{a b c}, F_{a b c}\right\rangle_{\text {Bound-Bound }},
$$

where the subscript "Free-Free" indicates the mutual interaction of two free electrons, "Free-Bound" denotes the mutual interaction of free and bound electrons, and "BoundBound" denotes the mutual interaction of two bound electrons. Thus, using the definitions (2.5), we can calculate the different terms of (2.3) in terms of couplings of parallel and anti-parallel spins [18]. The corresponding expressions are shown in Appendix A.

In summary, from the above analysis, by breaking pairs into electrons, standard Dirac equations are obtained, and hence, the relation between gauge fields and their sources, i.e. fermions, becomes clear. These results are very similar to those in M-theory [14, 15, 18]. Furthermore, one can use these expressions to show that, by joining electrons and forming a pair, 2-form gauge fields are created: these gauge fields play the role of graviton tensor modes between two electrons $\left(\Psi^{\dagger a, U}\left\langle F_{a b c}, F^{i^{\prime} b c}\right\rangle \psi_{i^{\prime}}^{L}\right)$. Specifically, one can obtain the couplings between electrons in terms of curvature of graphene. As it was shown in $[14,15,18,35,36]$, the metric can be antisymmetric, since, for instance, M1-branes are linked to anti-M1-branes and form a new system [14, 15, 18]. Thus, for this system the metric can be constructed from metrics of two M1's as:

Metric of system $\equiv(\text { Metric M1 })_{1} \otimes(\text { Metric M1 })_{2}-(\text { Metric M1 })_{2} \otimes(\text { Metric M1 })_{1}$

and therefore it can be antisymmetric. On the other hand, the graviton tensor mode has a direct relation with the metric and it can be anti-symmetric.

The same conditions can be realized in the graphene. In particular, the metric in a graphene can be constructed from the metric of pairs, such as for two electrons of a pair:

$$
\begin{aligned}
\text { Metric of pair } \equiv & (\text { Metric electron })_{1} \otimes(\text { Metric electron })_{2} \\
& -(\text { Metric electron })_{2} \otimes(\text { Metric electron })_{1} .
\end{aligned}
$$

Since this metric can be antisymmetric, the tensor mode of the graviton may be antisymmetric. Hence, the 2-form gauge fields have a direct relation with the graviton, and also with the metric of pairs in the graphene. 
Using the aforementioned method in a graphene structure, we can obtain the relation between fermions and curvatures $[14,15,18]$ :

$$
\begin{aligned}
A^{a b}= & g^{a b}=h^{a b}=h_{1}^{a b^{\prime}} \otimes h_{2}^{b^{\prime} b}-h_{2}^{b b^{\prime}} \otimes h_{1}^{a b^{\prime}} \\
F_{a b c}= & \partial_{a} A_{b c}-\partial_{b} A_{c a}+\partial_{c} A_{a b}=2\left(\partial_{\mu} g_{\nu \lambda}+\partial_{\nu} g_{\mu \lambda}-\partial_{\lambda} g_{\mu \nu}\right)=2 \Gamma_{\mu \nu \lambda} \\
\left\langle F_{\sigma \lambda}^{\rho}, F^{\lambda}{ }_{\mu \nu}\right\rangle= & \left\langle\left[X^{\rho}, X_{\sigma}, X_{\lambda}\right],\left[X^{\lambda}, X_{\mu}, X_{\nu}\right]\right\rangle \\
= & {\left[X_{\nu},\left[X^{\rho}, X_{\sigma}, X_{\mu}\right]\right]-\left[X_{\mu},\left[X^{\rho}, X_{\sigma}, X_{\nu}\right]\right] } \\
& +\left[X^{\rho}, X_{\lambda}, X_{\nu}\right]\left[X^{\lambda}, X_{\sigma}, X_{\mu}\right]-\left[X^{\rho}, X_{\lambda}, X_{\mu}\right]\left[X^{\lambda}, X_{\sigma}, X_{\nu}\right] \\
= & \partial_{\nu} \Gamma_{\sigma \mu}^{\rho}-\partial_{\mu} \Gamma_{\sigma \nu}^{\rho}+\Gamma_{\lambda \nu}^{\rho} \Gamma_{\sigma \mu}^{\lambda}-\Gamma_{\lambda \mu}^{\rho} \Gamma_{\sigma \nu}^{\lambda}=R_{\sigma \mu \nu}^{\rho},
\end{aligned}
$$

where $h^{a b}$ is the metric which is seen by the electron, $X_{\sigma}$ is the scalar which is created by pairing two anti-parallel electrons, and $R_{\sigma \mu \nu}^{\rho}$ is the Riemann tensor. Additionally, we obtain

$$
\left\langle F_{a b c}, F_{a^{\prime}}^{b c}\right\rangle=R_{a a^{\prime}}^{a n t i-\text { parallel }}-R_{a a^{\prime}}^{\text {parallel }},
$$

and

$$
\begin{aligned}
R_{M N}= & R_{a a^{\prime}}+R_{i a^{\prime}}+R_{i j^{\prime}}=R_{\text {Free-Free }}^{\text {anti-parallel }}+R_{\text {Free-Bound }}^{\text {anti-parallel }}+R_{\text {Bound-Bound }}^{\text {anti-parallel }} \\
& -R_{\text {Free-Free }}^{\text {parallel }}-R_{\text {Free-Bound }}^{\text {parallel }}-R_{\text {Bound-Bound }}^{\text {parallel }} .
\end{aligned}
$$

Finally, the term $\left\langle\partial^{b} \partial^{a} X^{i}, \partial_{b} \partial_{a} X^{i}\right\rangle$ is given in Appendix B. In the above expressions, $R_{\text {Bound-Bound }}^{\text {anti-parallel }}$ is the curvature produced by the interaction of two bound anti-parallel electrons, $R_{\text {Bound-Bound }}^{\text {parallel }}$ is the curvature created by interaction of two bound parallel electrons, $R_{\text {Free-Free }}^{\text {anti-parallel }}$ is the curvature produced by the interaction of two free anti-parallel electrons, $R_{\text {Free-Free }}^{\text {parallel }}$ is the curvature created by interaction of two free parallel electrons, $R_{\text {Free-Bound }}^{\text {anti-parallel }}$ is the curvature produced by the interaction of free and bound anti-parallel electrons and $R_{\text {Free-Free }}^{\text {parallel }}$ is the curvature created by interaction of free and bound parallel electrons.

In summary, from the above expressions, we deduce that there are two types of effective gravity: one related to parallel spins and one related to anti-parallel spins. Moreover, we have three types of curvatures: A first type of curvature is created by mutual coupling of free electrons. A second type of curvature is produced by coupling of free electrons to bound electrons. Lastly, a third type of curvature is produced by mutual coupling of bound electrons. Note that the curvature between anti-symmetric fermions is positive, while the curvature between parallel spins is negative. In fact, the gravity between antiparallel spins, namely $\Psi^{\dagger a, U} R_{a a^{\prime}}^{a n t i-p a r a l l e l} \Psi^{a^{\prime}, L}$, creates an attracting force, while the antigravity between parallel spins, i.e. $-\Psi^{\dagger a, U} R_{a a^{\prime}}^{\text {parallel }} \Psi^{a^{\prime}, U}$, produces a repelling force. This is explained since curvature has a direct relation with energy-momentum tensor, namely $\Psi^{\dagger a, U} R_{a a^{\prime}}^{\text {parallel }} \Psi^{a^{\prime}, U} \approx \Psi^{\dagger a, U} T_{a a^{\prime}}^{\text {parallel }} \Psi^{a^{\prime}, U}$. Since one of the components of this tensor is the momentum, i.e. $T_{a a^{\prime}} \propto P$, by changing the curvature of the system, the momentum changes and attractive or repulsive force emerge. Schematically we have:

$$
\begin{aligned}
& -\Psi^{\dagger a, U} R_{a a^{\prime}}^{\text {parallel }} \Psi^{a^{\prime}, U} \approx-\Psi^{\dagger a, U} T_{a a^{\prime}}^{\text {parallel }} \Psi^{a^{\prime}, U} \propto-\Psi^{\dagger a, U} \vec{P} \Psi^{a^{\prime}, U} \\
\Rightarrow & -\frac{d}{d t}\left(\Psi^{\dagger a, U} R_{a a^{\prime}}^{p a r a l l e l} \Psi^{a^{\prime}, U}\right) \approx-\frac{d}{d t}\left(\Psi^{\dagger a, U} \vec{P} \Psi^{a^{\prime}, U}\right) \approx-\Psi^{\dagger a, U} F \Psi^{a^{\prime}, U}+\cdots,
\end{aligned}
$$


and

$$
\begin{gathered}
\Psi^{\dagger a, U} R_{a a^{\prime}}^{\text {anti-parallel }} \Psi^{a^{\prime}, L} \approx \Psi^{\dagger a, U} T_{a a^{\prime}}^{\text {anti-parallel }} \Psi^{a^{\prime}, L} \propto \Psi^{\dagger a, U} \vec{P} \Psi^{a^{\prime}, L} \\
\Rightarrow \frac{d}{d t}\left(\Psi^{\dagger a, U} R_{a a^{\prime}}^{\text {anti-parallel }} \Psi^{a^{\prime}, L}\right) \approx \frac{d}{d t}\left(\Psi^{\dagger a, U} \vec{P} \Psi^{a^{\prime}, L}\right) \approx \Psi^{\dagger a, U} F \Psi^{a^{\prime}, L}+\cdots .
\end{gathered}
$$

From the above analysis, we deduce that positive curvature between anti-parallel spins leads to an attractive force between them, while negative curvature between parallel spins causes a mutual repulsive force. These forces produce the current density in the system, which we are interested in calculating.

Let us start by considering the interaction between electrons of one atom with those of neighbor atoms, and then we generalize it to the total system. Substituting expressions (2.12) and (B.1) in Eq. (2.3), for one atom of graphene $(n=1)$, after some algebra, we extract the energy as:

$$
\begin{aligned}
& E_{\text {system }}=\int d^{4} x \rho \\
& =\int d^{4} x \sqrt{-g}\left\{-\left(1-m_{g}^{2}\right)\left[\left(R_{\text {Free-Free }}^{\text {parallel }}\right)^{2}+\left(R_{\text {Free-Free }}^{\text {anti-parallel }}\right)^{2}+\left(R_{\text {Free-Bound }}^{\text {parallel }}\right)^{2}\right.\right. \\
& +\left(R_{\text {Free-Bound }}^{\text {anti-parallel }}\right)^{2}+\left(R_{\text {Bound-Bound }}^{\text {parallel }}\right)^{2}+\left(R_{\text {Bound-Bound }}^{\text {anti-parallel }}\right)^{2} \\
& +\left(R_{\text {Free-Free }}^{\text {parallel }} R_{\text {Free-Free }}^{\text {anti-parallel }}\right) \partial^{2}\left(R_{\text {Free-Free }}^{\text {parallel }}+R_{\text {Free-Free }}^{\text {anti-parallel }}\right) \\
& +\left(R_{\text {Free-Bound }}^{\text {parallel }} R_{\text {Free-Bound }}^{\text {anti-parallel }}\right) \partial^{2}\left(R_{\text {Free-Bound }}^{\text {parallel }}+R_{\text {Free-Bound }}^{\text {anti-parallel }}\right) \\
& \left.+\left(R_{\text {Bound-Bound }}^{\text {parallel }} R_{\text {Bound-Bound }}^{\text {anti-parallel }}\right) \partial^{2}\left(R_{\text {Bound-Bound }}^{\text {parallel }}+R_{\text {Bound-Bound }}^{\text {anti-parallel }}\right)\right] \\
& +m_{g}^{2} \lambda^{2} \delta_{\rho_{1} \sigma_{1}}^{\mu_{1} \nu_{1}}\left[R_{\text {Free-Free, } \mu_{1} \nu_{1}}^{\text {anti-parallel, } \sigma_{1} \sigma_{1}}+R_{\text {Bound-Bound, } \mu_{1} \nu_{1}}^{\text {anti-parallel }, \rho_{1} \sigma_{1}}+R_{\text {Free-Bound, } \mu_{1} \nu_{1}}^{\text {anti-parallel, } \rho_{1} \sigma_{1}}\right. \\
& \left.\left.-R_{\text {Free-Free, } \mu_{1} \nu_{1}}^{\text {parallel, } \rho_{1} \sigma_{1}}+R_{\text {Bound-Bound, } \mu_{1} \nu_{1}}^{\text {parallel, } \rho_{1} \sigma_{1}}+R_{\text {Free-Bound }, \mu_{1} \nu_{1}}^{\text {parallel, } \rho_{1} \sigma_{1}}\right]\right\},
\end{aligned}
$$

where $m_{g}^{2}=(\lambda)^{2} \operatorname{det}\left(\left[X_{\alpha}^{j} T^{\alpha}, X_{\beta}^{k} T^{\beta}, X_{\gamma}^{k^{\prime}} T^{\gamma}\right]\right)$ is the graviton mass square. Note that, for the sake of simplicity, we have chosen

$$
\Psi^{\dagger a, L} \psi_{a}^{U}=\Psi^{\dagger a, U} \psi_{a}^{L}=\Psi^{\dagger a, L} \Psi_{a}^{U}=\Psi^{\dagger a, U} \Psi_{a}^{L}=\psi^{\dagger a, U} \psi_{a}^{L}=\psi^{\dagger a, L} \psi_{a}^{U}=l_{1},
$$

and

$$
\Psi^{\dagger a, U} \psi_{a}^{U}=\Psi^{\dagger a, L} \psi_{a}^{L}=\Psi^{\dagger a, U} \Psi_{a}^{U}=\Psi^{\dagger a, L} \Psi_{a}^{L}=\psi^{\dagger a, U} \psi_{a}^{U}=\psi^{\dagger a, L} \psi_{a}^{L}=l_{2},
$$

where $l_{1}$ is the coupling between two anti-parallel spins and $l_{2}$ the coupling between parallel spins. Under these considerations, we can calculate the curvatures as

$$
\begin{aligned}
& R_{\text {Free-Free }}^{\text {parallel }}=R_{\text {Free-Bound }}^{\text {parallel }}=R_{\text {Bound-Bound }}^{\text {parallel }} \approx l_{2}-l_{2}^{\prime} \\
& R_{\text {Free-Free }}^{\text {anti-parallel }}=R_{\text {Free-Ballel }}^{\text {anti-pound }}=R_{\text {Bound-Bound }}^{\text {anti-paralle }} \approx l_{1}+l_{1}^{\prime} .
\end{aligned}
$$

Thus, using these useful relations, together with (2.15) and the chain rule, we obtain

$$
\frac{\partial E_{\text {system }}}{\partial t}=\frac{\partial E_{\text {system }}}{\partial l_{1}} \frac{\partial l_{1}}{\partial t}+\frac{\partial E_{\text {system }}}{\partial l_{2}} \frac{\partial l_{2}}{\partial t} .
$$


Finally, we get the current density as:

$$
\begin{aligned}
& I=\int d^{4} x J \approx \frac{\partial E_{\text {system }}}{\partial t} \\
& \approx-\int d^{4} x \sqrt{-g}\left\{-\left(1-m_{g}^{2}\right)\left[\left(R_{\text {Free-Free }}^{\text {parallel }}\right)^{2}-\left(R_{\text {Free-Free }}^{\text {anti-parallel }}\right)^{2}+\left(R_{\text {Free-Bound }}^{\text {parallel }}\right)^{2}\right.\right. \\
& -\left(R_{\text {Free-Bound }}^{\text {anti-parallel }}\right)^{2}+\left(R_{\text {Bound-Bound }}^{\text {parallel }}\right)^{2}-\left(R_{\text {Bound-Bound }}^{\text {anti-parallel }}\right)^{2} \\
& +\left(R_{\text {Free-Free }}^{\text {parallel }} R_{\text {Free-Free }}^{\text {anti-parallel }}\right) \partial^{2}\left(R_{\text {Free-Free }}^{\text {parallel }}-R_{\text {Free-Free }}^{\text {anti-parallel }}\right) \\
& +\left(R_{\text {Free-Bound }}^{\text {parallel }} R_{\text {Free-Bound }}^{\text {anti-parallel }}\right) \partial^{2}\left(R_{\text {Free-Bound }}^{\text {parallel }}-R_{\text {Free-Bound }}^{\text {anti-parallel }}\right) \\
& \left.+\left(R_{\text {Bound-Bound }}^{\text {parallel }} R_{\text {Bound-Bound }}^{\text {anti-parallel }}\right) \partial^{2}\left(R_{\text {Bound-Bound }}^{\text {parallel }}-R_{\text {Bound-Bound }}^{\text {anti-parallel }}\right)\right] \\
& +m_{g}^{2} \lambda^{2} \delta_{\rho_{1} \sigma_{1}}^{\mu_{1} \nu_{1}}\left[R_{\text {Free-Free }, \mu_{1} \nu_{1}}^{\text {anti-paralle }, \rho_{1} \sigma_{1}}+R_{\text {Bound }- \text { Bound, } \mu_{1} \nu_{1}}^{\text {anti-parallel, } \rho_{1} \sigma_{1}}+R_{\text {Free-Bound, } \mu_{1} \nu_{1}}^{\text {anti-parallel } \rho_{1} \sigma_{1}}\right. \\
& \left.\left.+R_{\text {Free-Free }, \mu_{1} \nu_{1}}^{\text {parallel, } \rho_{1} \sigma_{1}}+R_{\text {Bound }- \text { Bound, } \mu_{1} \nu_{1}}^{\text {parallel } \rho_{1} \sigma_{1}}+R_{\text {Free-Bound, } \mu_{1} \nu_{1}}^{\text {parallel, } \rho_{1} \sigma_{1}}\right]\right\} \text {. }
\end{aligned}
$$

From this expression, it is easy to see that the current density in graphene depends on the curvature produced by parallel and anti-parallel spins. For a symmetric graphene, curvatures of parallel spins are canceled by curvatures of anti-parallel spins, and therefore the current density of the system decreases. Hence, by increasing the symmetry conductivity decreases.

Finally, in order to calculate the total current density in graphene, we should sum over currents in each atom, namely

$$
\vec{J}_{\text {system }}=\Sigma_{n=1}^{U} \delta_{b_{1} b_{2} \ldots b_{n}}^{a_{1}, a_{2} \ldots a_{n}} \vec{J}_{a_{1}}^{b_{1}} \ldots \vec{J}_{a_{n}}^{b_{n}}
$$

where $\vec{J}_{a_{n}}^{b_{n}}=\vec{J} \delta_{a_{n}}^{b_{n}}$. This expression indicates that the total current density in graphene depends on the curvatures of parallel spins and anti-parallel spins in each atom. If the current density in one atom is zero, electrons stop at that point and the total current density of the system becomes zero. This fact leads to the disappearance of conductivity. Thus, by breaking the symmetry in graphene, curvatures are created. This phenomenon leads to the production of current density and hence of conductivity in the system.

\section{The current density in graphene in presence of various defects}

In this section, we investigate the model in the specific case of standard graphene, and, additionally, considering heptagonal and pentagonal defects. In particular, using the definitions for couplings of parallel spins $\left(l_{2}\right)$ and for couplings of anti-parallel spins $\left(l_{1}\right)$, and substituting expressions (2.12) and (B.1) into expression (2.3), we obtain the following action for one atom in the graphene:

$$
\begin{aligned}
S_{c o-a t o m} \approx V \int d \cos \theta \Sigma_{n=1}^{p} & \left\{6 m_{g}^{2} \lambda^{2}\left[l_{1}-l_{2}-l_{1}^{\prime}+l_{2}^{\prime}+\left(l_{1}^{\prime}\right)^{2}-\left(l_{2}^{\prime}\right)^{2}\right]\right. \\
& \left.-3\left(1-m_{g}^{2}\right)\left[2 l_{1}^{2}+2 l_{2}^{2}+2\left(l_{1}^{\prime}\right)^{2}+2\left(l_{2}^{\prime}\right)^{2}+l_{1}^{2} l_{2}^{2}\left(l_{1}^{2}+l_{2}^{2}\right)^{\prime \prime}\right]\right\}^{\frac{1}{2}},
\end{aligned}
$$


with $V$ is the atom volume, and where we have assumed that the couplings depend only on $\theta$, i.e. the angle between two electrons in one graphene atom with respect to the centre of the atom, with' denoting derivative with respect to $\cos \theta$.

The variation of the action (3.1) provides the equations of motion as:

$$
\begin{gathered}
\left\{m _ { g } ^ { 2 } l _ { 1 } ^ { \prime } [ \lambda ^ { 2 } - ( 1 + 2 l _ { 1 } ^ { 2 } l _ { 2 } ^ { 2 } ) ] \left\{6 m_{g}^{2} \lambda^{2}\left[l_{1}-l_{2}-l_{1}^{\prime}+l_{2}^{\prime}+\left(l_{1}^{\prime}\right)^{2}-\left(l_{2}^{\prime}\right)^{2}\right]\right.\right. \\
\left.\left.-3\left(1-m_{g}^{2}\right)\left[2 l_{1}^{2}+2 l_{2}^{2}+2\left(l_{1}^{\prime}\right)^{2}+2\left(l_{2}^{\prime}\right)^{2}+l_{1}^{2} l_{2}^{2}\left(l_{1}^{2}+l_{2}^{2}\right)^{\prime \prime}\right]\right\}^{-\frac{1}{2}}\right\}^{\prime}= \\
\left\{\left(1-m_{g}^{2}\right) l_{1}\left[1+3 l_{2}^{2}\left(l_{1}^{2}+l_{2}^{2}\right)^{\prime \prime}\right]+m_{g}^{2} \lambda^{2}\right\}\left\{6 m_{g}^{2} \lambda^{2}\left[l_{1}-l_{2}-l_{1}^{\prime}+l_{2}^{\prime}+\left(l_{1}^{\prime}\right)^{2}-\left(l_{2}^{\prime}\right)^{2}\right]\right. \\
\left.-3\left(1-m_{g}^{2}\right)\left[2 l_{1}^{2}+2 l_{2}^{2}+2\left(l_{1}^{\prime}\right)^{2}+2\left(l_{2}^{\prime}\right)^{2}+l_{1}^{2} l_{2}^{2}\left(l_{1}^{2}+l_{2}^{2}\right)^{\prime \prime}\right]\right\}^{-\frac{1}{2}}, \\
\left\{l _ { 2 } ^ { \prime } [ ( 1 - m _ { g } ^ { 2 } ) ( 1 - 2 l _ { 1 } ^ { 2 } l _ { 2 } ^ { 2 } ) - m _ { g } ^ { 2 } \lambda ^ { 2 } ] \left\{6 m_{g}^{2} \lambda^{2}\left[l_{1}-l_{2}-l_{1}^{\prime}+l_{2}^{\prime}+\left(l_{1}^{\prime}\right)^{2}-\left(l_{2}^{\prime}\right)^{2}\right]\right.\right. \\
\left.\left.\quad-3\left(1-m_{g}^{2}\right)\left[2 l_{1}^{2}+2 l_{2}^{2}+2\left(l_{1}^{\prime}\right)^{2}+2\left(l_{2}^{\prime}\right)^{2}+l_{1}^{2} l_{2}^{2}\left(l_{1}^{2}+l_{2}^{2}\right)^{\prime \prime}\right]\right\}^{-\frac{1}{2}}\right\}^{\prime}= \\
\left\{\left(1-m_{g}^{2}\right) l_{2}\left[1-3 l_{1}^{2}\left(l_{1}^{2}+l_{2}^{2}\right)^{\prime \prime}\right]-m_{g}^{2} \lambda^{2}\right\}\left\{6 m_{g}^{2} \lambda^{2}\left[l_{1}-l_{2}-l_{1}^{\prime}+l_{2}^{\prime}+\left(l_{1}^{\prime}\right)^{2}-\left(l_{2}^{\prime}\right)^{2}\right]\right. \\
\left.-3\left(1-m_{g}^{2}\right)\left[2 l_{1}^{2}+2 l_{2}^{2}+2\left(l_{1}^{\prime}\right)^{2}+2\left(l_{2}^{\prime}\right)^{2}+l_{1}^{2} l_{2}^{2}\left(l_{1}^{2}+l_{2}^{2}\right)^{\prime \prime}\right]\right\}^{-\frac{1}{2}} .
\end{gathered}
$$

One can easily find the approximate solution of these equations as:

$$
\begin{aligned}
& l_{1} \approx \cos \left(\theta_{1}\right) \\
& l_{2} \approx \cos \left(\theta_{2}\right)=\left(1-m_{g}^{2}\right) \cos \left(\theta_{1}\right)-m_{g}^{2} \lambda^{2} \sin \left(\theta_{1}\right),
\end{aligned}
$$

and therefore expressions $(2.16),(2.17)$ lead to

$$
\Psi=\psi \approx \sqrt{\cos (\theta)} .
$$

Solutions (3.4),(3.5) allow us to calculate the curvatures through (2.18), and finally the current density through (2.20).

\subsection{Graphene without defects (hexagonal)}

Let us first study the simple case of hexagonal graphene. It has been shown that, in the graphene, the difference between angles of couplings of parallel spins and anti-parallel spins is around $\frac{\pi}{3}$ [37-39], a result that is in agreement with the symmetries of the graphene. In each hexagonal graphene molecule, the electrons of each atom should be anti-parallel with respect to the electrons of neighbor atoms, and therefore the angle between them with respect to the molecule center is $\frac{\pi}{3}$. On the other hand, the angle between parallel spins is $\frac{2 \pi}{3}$. Thus, the difference between angles of couplings of parallel spins and anti-parallel spins is $\frac{\pi}{3}$. Hence, choosing the parameter values $m_{g}^{2}=\frac{1}{2}$ and $m_{g}^{2} \lambda^{2}=\sqrt{3}$, we get

$$
\begin{aligned}
& l_{1} \approx \cos \left(\theta_{1}\right) \\
& l_{2} \approx \cos \left(\theta_{1}+\frac{\pi}{3}\right),
\end{aligned}
$$

which is exactly this realization. 
In order to calculate the action of conductivity in the graphene, we should first obtain the values of the curvature tensor at the places of the atoms. Each carbon has three bound electrons and one free electron, where the bound states are located along three axes that form an angle of $2 \pi / 3$. The angle between two electron spins has a direct relation to the radius of the hexagonal molecule $(R)$ and the separation distance between two electrons $(L)\left(\theta=\frac{L}{R}\right.$ in radians). Thus, if we calculate the curvature in terms of the angle, we can obtain the explicit form of it in terms of the other parameters of the graphene. We assume that one axis lies along $X$ (see Fig. 1), and additionally we consider that the couplings for free and bound electrons are the same. Thus, we obtain the couplings of parallel and anti-parallel spins in terms of their angle with respect to the $X$-axis, namely

$$
\begin{aligned}
& l_{1}^{1-1} \approx \cos (0)=1, \quad l_{1}^{1-2} \approx \cos (2 \pi / 3)=-\frac{1}{2}, \quad l_{1}^{1-3} \approx \cos (4 \pi / 3)=-\frac{1}{2}, \\
& \left(l_{1}^{\prime}\right)^{1-1} \approx \sin (0)=0, \quad\left(l_{1}^{\prime}\right)^{1-2} \approx \sin (2 \pi / 3)=\frac{\sqrt{3}}{2}, \quad\left(l_{1}^{\prime}\right)^{1-3} \approx \sin (4 \pi / 3)=-\frac{\sqrt{3}}{2},
\end{aligned}
$$

and

$$
\begin{aligned}
& l_{2}^{1-1} \approx \cos (\pi / 3)=\frac{1}{2}, \quad l_{2}^{1-2} \approx \cos (\pi)=-1, \quad l_{2}^{1-3} \approx \cos (5 \pi / 3)=\frac{1}{2}, \\
& \left(l_{2}^{\prime}\right)^{1-1} \approx \sin (\pi / 3)=\frac{\sqrt{3}}{2}, \quad\left(l_{2}^{\prime}\right)^{1-2} \approx \sin (\pi)=0, \quad\left(l_{2}^{\prime}\right)^{1-3} \approx \sin (5 \pi / 3)=-\frac{\sqrt{3}}{2} .
\end{aligned}
$$

Substituting these values into expressions (2.6) and (2.12), we can calculate the curvatures

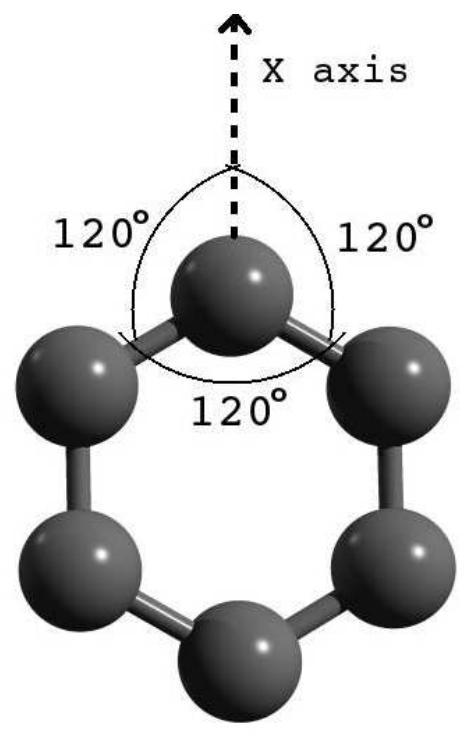

Figure 1. The angle between electron pairs in the graphene without defects (hexagonal).

for parallel and anti-parallel spins as

$$
\begin{aligned}
& R_{\text {Free/Bound-Free/Bound }}^{\text {anti-parallel }}=l_{1}^{1-1}+l_{1}^{1-2}+l_{1}^{1-3}+\left(l_{1}^{\prime}\right)^{1-1}+\left(l_{1}^{\prime}\right)^{1-2}+\left(l_{1}^{\prime}\right)^{1-3}=0 \\
& R_{\text {Frae/Bound-Free/Bound }}^{\text {parallel }}=l_{2}^{1-1}+l_{2}^{1-2}+l_{2}^{1-3}-\left(l_{2}^{\prime}\right)^{1-1}-\left(l_{2}^{\prime}\right)^{1-2}-\left(l_{2}^{\prime}\right)^{1-3}=0,
\end{aligned}
$$


and then inserting into (2.20) for the current density we obtain

$$
J \approx 0 .
$$

Hence, we conclude that for the standard graphene without defects, the current density is zero and thus the electrons do not collectively move in any specific direction. Consequently, electron moves randomly and superconductivity disappears.

\subsection{Graphene with heptagonal defects}

Let us now investigate the case of graphene with heptagonal defects. Similar to hexagonal case, we assume one axis along $X$ (see Fig. 2), and we consider that the couplings for free and bound electrons are the same. We calculate the couplings of parallel and anti-parallel spins in terms of their angles with respect to the $X$-axis as:

$$
\begin{aligned}
& l_{1}^{1-1} \approx \cos (0)=1, \quad l_{1}^{1-2} \approx \cos (116)=-0.434, \quad l_{1}^{1-3} \approx \cos (244)=-0.434, \\
& \left(l_{1}^{\prime}\right)^{1-1} \approx \sin (0)=0, \quad\left(l_{1}^{\prime}\right)^{1-2} \approx \sin (116)=0.901, \quad\left(l_{1}^{\prime}\right)^{1-3} \approx \sin (244)=-0.901,
\end{aligned}
$$

and

$l_{2}^{1-1} \approx \cos (60)=\frac{1}{2}, \quad l_{2}^{1-2} \approx \cos (176)=-0.997, \quad l_{2}^{1-3} \approx \cos (304)=0.563$,

$\left(l_{2}^{\prime}\right)^{1-1} \approx \sin (60)=0.866, \quad\left(l_{2}^{\prime}\right)^{1-2} \approx \sin (116)=0.075, \quad\left(l_{2}^{\prime}\right)^{1-3} \approx \sin (304)=-0.826 .(3.12)$

Substituting these values into (2.6) and (2.12), we can calculate the curvatures for parallel

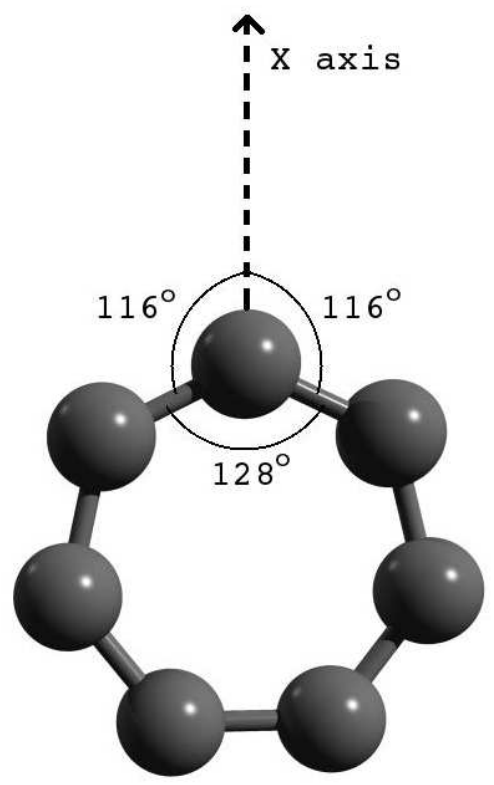

Figure 2. The angle between electron pairs in the graphene with a heptagonal defect.

and anti-parallel spins as

$$
\begin{aligned}
& R_{\text {Free/Bound-Free/Bound }}^{\text {anti-parallel }}=l_{1}^{1-1}+l_{1}^{1-2}+l_{1}^{1-3}+\left(l_{1}^{\prime}\right)^{1-1}+\left(l_{1}^{\prime}\right)^{1-2}+\left(l_{1}^{\prime}\right)^{1-3}=0.132 \\
& R_{\text {Free/Bound-Free/Bound }}^{\text {parallel }}=l_{2}^{1-1}+l_{2}^{1-2}+l_{2}^{1-3}-\left(l_{2}^{\prime}\right)^{1-1}-\left(l_{2}^{\prime}\right)^{1-2}-\left(l_{2}^{\prime}\right)^{1-3}=-0.048 .(3.1
\end{aligned}
$$


Note that the positive sign for anti-parallel spins implies that the electrons are attracted by electrons of neighbor molecules and that the coupling of anti-parallel spins is along the $X$-axis, while the negative sign for parallel spins means that electrons are repelled by parallel spins in neighbor molecules and that the coupling of parallel spins is along the negative $X$-axis. Substituting the values (3.13) into the current density (2.20), we obtain

$$
J \approx 0.458
$$

Since the current density is positive, we deduce that electrons are repelled by neighbor molecules and move along the $X$-axis (the curvature produced by parallel spins is larger than the curvature produced by anti-parallel spins and therefore a negative force is applied to electrons and they move in opposite directions with respect to the molecule). This result is also in agreement with previous predictions that the curvature of heptagonal defect is negative [13, 37-39].

\subsection{Graphene with pentagonal defects}

Finally, let us investigate the case of pentagonal defects. Similarly to the previous cases, we consider one axis along $X$ (see Fig. 3), and we assume that the couplings for free and bound electrons are the same. We calculate the couplings of parallel and anti-parallel spins in terms of their angles with respect to the $X$-axis as:

$$
\begin{aligned}
& l_{1}^{1-1} \approx \cos (0)=1, \quad l_{1}^{1-2} \approx \cos (126)=-0.587, \quad l_{1}^{1-3} \approx \cos (234)=-0.587 \\
& \left(l_{1}^{\prime}\right)^{1-1} \approx \sin (0)=0, \quad\left(l_{1}^{\prime}\right)^{1-2} \approx \sin (126)=0.809, \quad\left(l_{1}^{\prime}\right)^{1-3} \approx \sin (234)=-0.809
\end{aligned}
$$

$l_{2}^{1-1} \approx \cos (60)=\frac{1}{2}, \quad l_{2}^{1-2} \approx \cos (186)=-0.994, \quad l_{2}^{1-3} \approx \cos (294)=0.406$,

$\left(l_{2}^{\prime}\right)^{1-1} \approx \sin (60)=0.866,\left(l_{2}^{\prime}\right)^{1-2} \approx \sin (186)=-0.104,\left(l_{2}^{\prime}\right)^{1-3} \approx \sin (294)=-0.913$.

Substituting these values into expressions (2.6) and (2.12), we can calculate the curvatures for parallel and anti-parallel spins as

$$
\begin{aligned}
& R_{\text {Free/Bound-Free/Bound }}^{\text {anti-parallel }}=l_{1}^{1-1}+l_{1}^{1-2}+l_{1}^{1-3}+\left(l_{1}^{\prime}\right)^{1-1}+\left(l_{1}^{\prime}\right)^{1-2}+\left(l_{1}^{\prime}\right)^{1-3}=-0.174 \\
& R_{\text {Free/Bound-Free/Bound }}^{\text {parallel }}=l_{2}^{1-1}+l_{2}^{1-2}+l_{2}^{1-3}-\left(l_{2}^{\prime}\right)^{1-1}-\left(l_{2}^{\prime}\right)^{1-2}-\left(l_{2}^{\prime}\right)^{1-3}=0.063 .
\end{aligned}
$$

Substituting these values into the current density (2.20) we obtain

$$
J \approx-0.539
$$

The negative value of the current density implies that the electrons are absorbed by pentagonal defects and move along the negative $X$-axis, i.e this type of defects induces a force to the free electrons and leads them to move towards the molecule. Hence, increasing the number of defects, the current density increases and the graphene tends to be a superconductor. In Fig. 4, we depict the dependence of the current density on different number of pentagonal defects. 


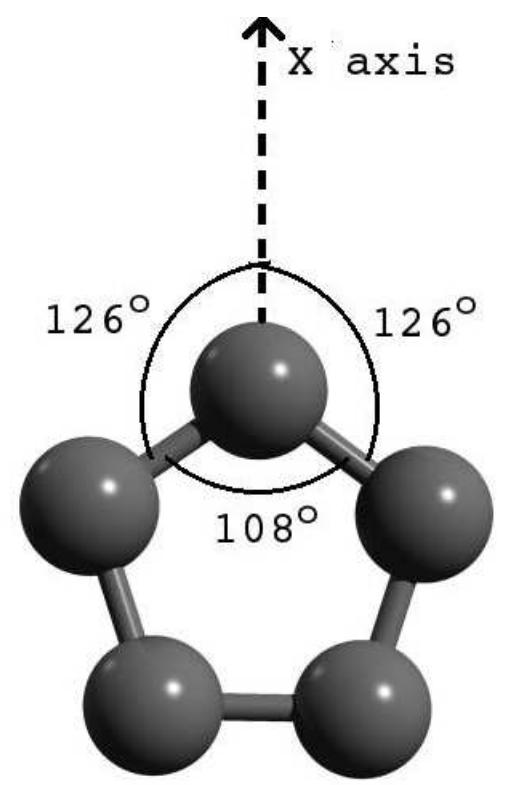

Figure 3. The angle between electron pairs in the graphene with a pentagonal defect.

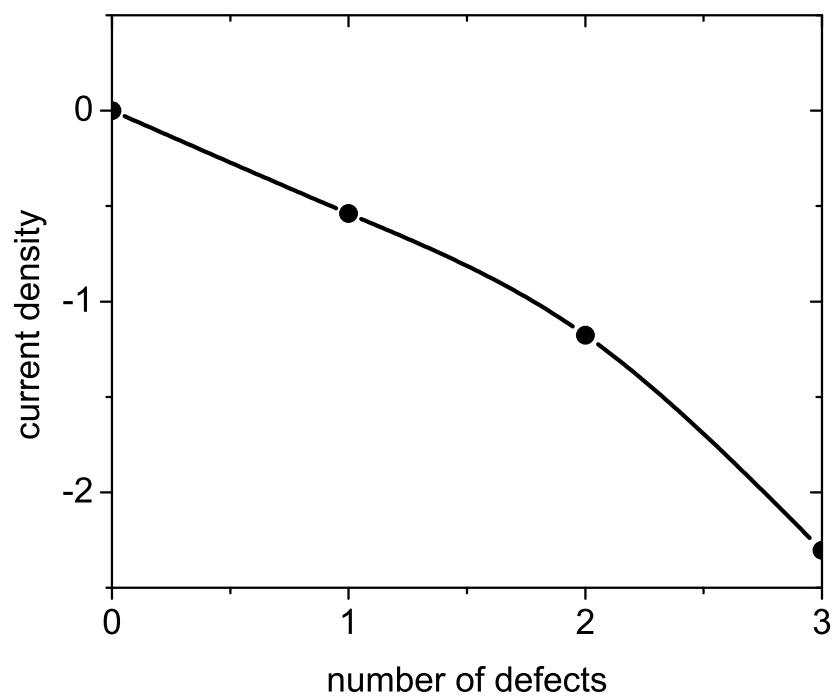

Figure 4. The dependence of the current density on the number of pentagonal defects.

In summary, in this section we found that heptagonal defects repel electrons while pentagonal defects absorb electrons. This is a consequence of the fact that in heptagonal defects the curvature of parallel spins is larger than the curvature produced by anti-parallel spins, while, in pentagonal defects, the curvature produced by parallel spins is smaller than the curvature produced by anti-parallel spins. These results are in agreement with previous analyses for defects in the graphene [13, 37-39]. 


\section{The current density in a graphene wormhole}

Let us consider the formalism and methods of the previous section in the case of a graphene wormhole. Such an object is created when the structure of the plain graphene is disrupted by the presence of heptagonal defects [37-39]. In the following, without loss of generality, we take into account the case of 12 defects, and in Fig. 5, we give a picture of such a graphene wormhole. The graphene wormhole consists of the upper and lower graphene

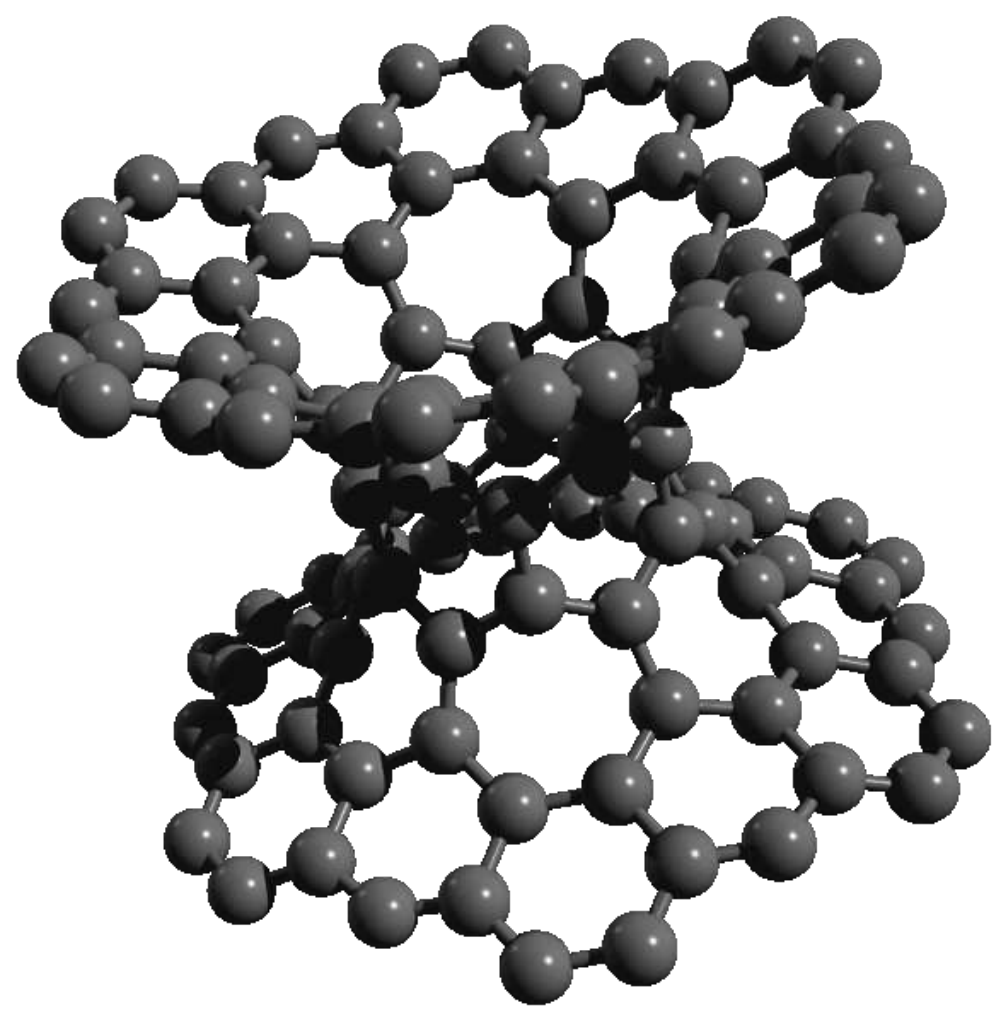

Figure 5. Graphene wormhole with 12 heptagonal defects.

sheets, each one connected to a wormhole bridge which is connected to the common connecting nanotube, as can be seen in Fig. 6 .

Let us now calculate the current density for this case. According to the results of the previous section, the current density should be zero in both graphene sheets and positive $(J \approx 0.458)$ in the case of a heptagonal defect. Additionally, we expect that, in the place of the wormhole bridges where 6 defects appear (and hence 12 in total, if we consider both sides of the connecting nanotube), the overall current density should be proportional to the current density for 1 defect, namely

$$
J \approx 6 \cdot 0.458=2.748
$$

The crucial question from the experimental point of view is what value of the current density would be measured in the region around the middle of the connecting nanotube. One would 


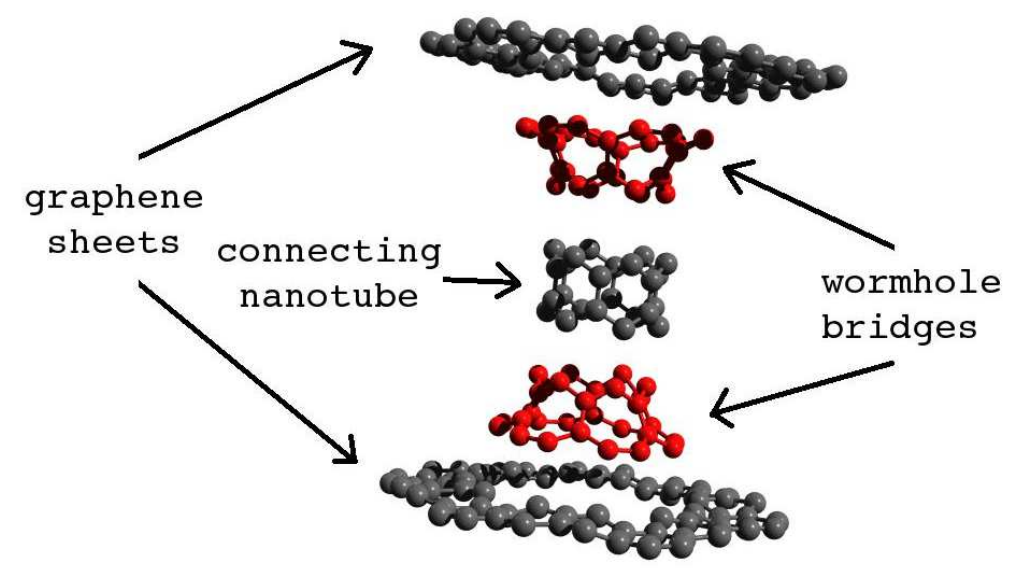

Figure 6. A schematic split of a graphene wormhole with 12 heptagonal defects.

expect that exactly at the middle, the current densities coming from opposite directions eliminate each other, and thus the overall current density is zero. On the other hand, at small distances away from the middle, the geometry of the structure differs significantly from the geometry in the wormhole bridge, and, therefore, one should expect a change of the current density. Hence, we can apply the procedure of the previous section in order to explicitly calculate the exact value.

In order to perform the calculation, we need the appropriate value of the angle $\theta_{1}$, substitute it into the expressions (3.7),(3.8) for the couplings $l_{1}, l_{2}$, and calculate the curvatures of the parallel and anti-parallel spins using (2.6) and (2.12). Finally, the current density is calculated using (2.20).

The value of $\theta_{1}$ depends on the distance from the defects: at shorter distances the character of the physical quantities is either the same as in the case of a simple defect, or it is the superposition of $n$ defects, respectively. At larger distances from the place of $n$ heptagonal defects, the geometry is approaching the case of the structure with 1 defect consisting of $6+n$ vortices, as can be seen from Fig. 7. Hence, we deduce that at large distances from the wormhole bridges, the value of the current density corresponds to the case of a dodecagon $(12=6+n$, where $n=6$ for each of the two parts of the wormhole structure). In this case, the value of $\theta_{1}$ corresponds to Fig. $7 \mathrm{~b}$, namely $\theta_{1}=105$. Thus, (3.7),(3.8) lead to

$$
\begin{aligned}
& l_{1}^{1-1} \approx \cos (0)=1, \quad l_{1}^{1-2} \approx \cos (105)=-0.259, \quad l_{1}^{1-3} \approx \cos (255)=-0.259, \\
& \left(l_{1}^{\prime}\right)^{1-1} \approx \sin (0)=0, \quad\left(l_{1}^{\prime}\right)^{1-2} \approx \sin (105)=0.966, \quad\left(l_{1}^{\prime}\right)^{1-3} \approx \sin (255)=-0.966,
\end{aligned}
$$

and

$$
\begin{aligned}
& l_{2}^{1-1} \approx \cos (60)=\frac{1}{2}, \quad l_{2}^{1-2} \approx \cos (165)=-0.966, \quad l_{2}^{1-3} \approx \cos (315)=0.707 \\
& \left(l_{2}^{\prime}\right)^{1-1} \approx \sin (60)=\frac{\sqrt{3}}{2}, \quad\left(l_{2}^{\prime}\right)^{1-2} \approx \sin (165)=0.259, \quad\left(l_{2}^{\prime}\right)^{1-3} \approx \sin (315)=-0.707 .
\end{aligned}
$$




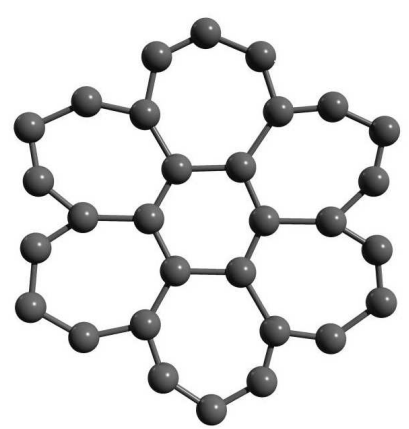

a) small distance

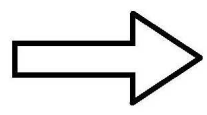

Figure 7. The geometric structure at small and large distance from the defects.

Substituting these values into expressions (2.6) and (2.12), we can calculate the curvatures for parallel and anti-parallel spins as

$$
\begin{aligned}
& R_{\text {Free/Bound-Free/Bound }}^{\text {anti-paralle }}=0.482, \\
& R_{\text {Fraee/Bound-Free/Bound }}^{\text {parallel }}=-0.177,
\end{aligned}
$$

and thus for the current density (2.20) we obtain

$$
J \approx 1.960 \text {. }
$$

Hence, we deduce that in the graphene wormhole, the current density is zero in the upper and lower graphene sheet, then it rises up to value 2.748 in the wormhole bridges, and in the connecting nanotube it decreases to the value 1.960. In the centre of the connecting nanotube, the current density is exactly zero due to the mutual elimination.

Let us close this section by mentioning that the number of defects in the graphene wormhole can differ from 2 to 12 defects, i.e. from 1 to 6 defects at each side. Therefore, the upper and lower sheets will not have the geometry of the plain graphene, and the corresponding current density will change, as well as the current density close to the middle of the connecting nanotube. In Fig. 8 we depict the dependence of the current density close to the middle of the connecting nanotube, on the number of defects.

\section{Summary and Discussion}

In this work we have studied, in the context of graphene structure, the exchange of gauge fields between electrons, which can be considered as a sort of effective gravitons. Such exchange leads to the emergence of conductivity. In this way, three types of curvature are produced, one between free electrons, one between bound electrons, and one between free and bound electrons. These fields create a sort of an effective gravity with positive curvature between parallel spins, and anti-gravity with negative curvature between parallel 


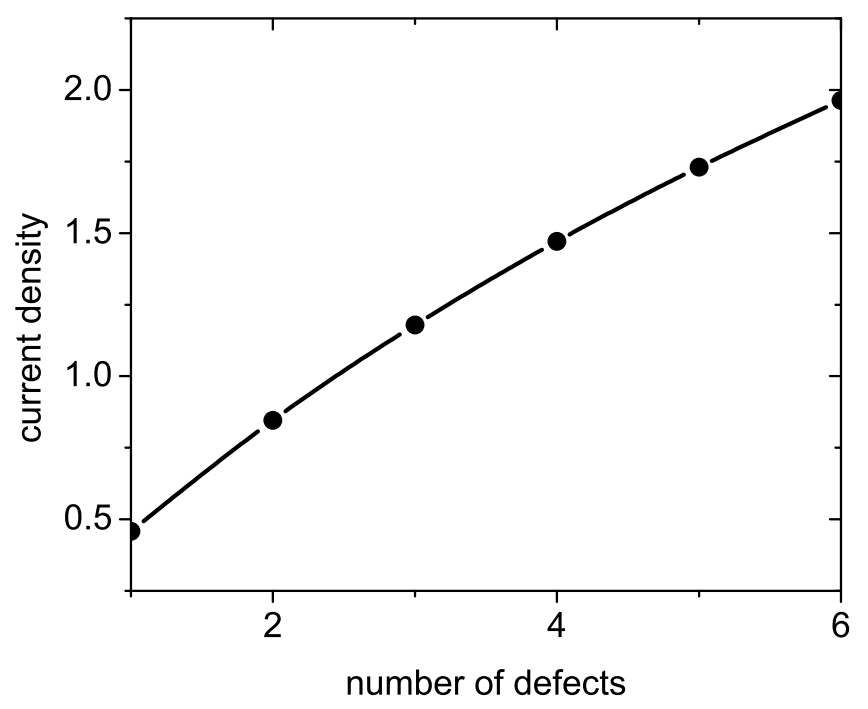

Figure 8. The dependence of the current density, close to the middle of the connecting nanotube, on the number of defects.

spins. The current density of free electrons has been obtained in terms of inequality between curvatures of parallel spins and those between anti-parallel spin

In particular, in Sec. 2, we obtained the current density in terms of curvature of parallel spins and anti-parallel spins and in Sec. 3 and 4, by substituting the relations between curvatures and parameters of graphene molecule, we have calculated the current density in terms of the angle between atoms with respect to the center of graphene molecule. In fact, in Sec. 2, using the concepts in M-theory, we introduced an action for conductivity in a graphene system in terms of gauge fields and fermions. Then, we obtained the relation between gauge fields and curvature of parallel spins and anti-parallel spins. Also, it has been shown that the curvature which is produced between parallel spins has an opposite sign with respect to the curvature which is generated between anti-parallel spins. These curvatures have a direct relation with the effective energy-momentum tensors. Also, changes in momentums have a direct relation with the applied force between spinors. Consequently, the force between parallel spin has an opposite sign with respect to the force between antiparallel spins as it is possible to observe in laboratory experiments. Using the relations between gauge fields, spinors and curvature, it is possible to obtain the energy of system in terms of difference between curvatures of parallel spins and anti-parallel spins. Besides, using this energy, the current density in terms of curvatures has been derived.

These results can be applied to realistic graphene structures and a relation between curvatures and graphene parameters, like the angles between atoms with respect to the molecular center, can be obtained. Using these relations, the current density, in terms of such angles, can be calculated. We have shown that, for the standard graphene with hexagonal molecules, the current density is zero and thus the electrons do not collectively 
move in any given direction. Consequently, electrons move randomly and superconductivity disappears. For graphene with heptagonal defects, since the current density is positive, it is possible to deduce that electrons are repelled by neighbor molecules and move outward from molecules (the curvature produced by parallel spins is larger than the curvature produced by anti-parallel spins and therefore a negative force is applied to electrons. As consequence, they move in opposite directions with respect to the molecule). This result is also in agreement with previous predictions that the curvature of heptagonal defect is negative [13, 37-39]. For graphene with pentagonal defects, the negative value of the current density implies that the electrons are absorbed by pentagonal defects and move towards molecules, i.e this type of defects induces a force to the free electrons and leads them to move towards the molecules. Hence, increasing the number of defects, the current density increases and the graphene tends to be a superconductor.

In Sec. 4, we deduced that, in the graphene wormhole, the current density is zero in the upper and lower graphene sheet, then it rises up to positive value in the wormhole bridges. On the other hand, in the connecting nanotube, it decreases to the lower values. In the centre of the connecting nanotube, the current density is exactly zero due to the mutual elimination.

In standard graphene, due to its symmetry, the curvature of anti-parallel spins is canceled by the curvature of parallel spins, and hence the total current density of free electrons becomes zero. Thus, the free electrons do not move in any special direction and therefore conductivity disappears. On the other hand, for some particular types of defects in the graphene, anti-parallel spins come closer mutually, their curvature increases, and a sort of modified gravity emerges. Consequently, the current density grows and conductivity increases. Similarly, for some other types of defects, parallel spins approach each other, their negative curvature increases and (modified) anti-gravity appears. In this case, the sign of current density reverses, the electrons move in opposite direction, and a new conductivity appears along this new direction.

In the case of more complicated structures, such as graphene wormholes, the current density in the different regions of the molecular surface has different values. Moreover, the curvature induced by the defects cannot be clearly determined from the type of the defects and it is given by the chemical structure of the whole molecule. As a result, the current density arising from these defects depends on the distance from them. We derived the current density for the graphene wormhole which includes 12 heptagonal defects, and we have found an approximation for the cases where the number of defects changes. The current density on the curved wormhole sheets in such modified structures remains an open question.

Furthermore, it is possible to show that each defect produces a given form of extended gravity. In particular, as reported in Appendix C, no defect, heptagonal defects and pentagonal defects give rise to different forms of $f(R)$ gravity.

In general, the type of defects can be determined by the chemical structure of the whole molecule. This means that each defect produces a different type of gravity and current density. For example, pentagonal molecules absorb electrons, while heptagonal molecules repel them. By inserting defects in graphene suitable places, electrons are repelled by some 
molecules and absorbed by other ones and move in an given direction. This helps us to design a graphene which conducts electrons in an given direction. In fact, for producing a good superconductor, it is needed to insert heptagonal and pentagonal molecules in suitable places between hexagonal graphene molecules. On the other hand, this means that the gravitational anologue picture can discriminate among the various graphene structures.

As final remark, it is worth noticing that, from an experimental point of view, these graphene defects structures could be probed using currents facilities and experimental apparatuses working in several laboratories $[40,41]$ considering that the presence of the absence of precise current densities are the final test beds for the models. On the other hand, analogue models of gravity [42] could be realized by graphene considering the 2 -form tensor fields discussed above. In a forthcoming paper, we will discuss in details possible experimental realizations of these structures.

\section{Acknowledgments}

The work by A. Sepehri has been financially supported by the Research Institute for Astronomy and Astrophysics of Maragha (RIAAM), Iran, under research project No.1/4165-14. The work was partly supported by VEGA Grant No. 2/0009/16. R. Pincak would like to thank the TH division in CERN for hospitality. This work was partially supported by the JSPS Grant-in-Aid for Young Scientists (B) \# 25800136 and the research-funds given by Fukushima University (K.B.). S. Capozziello acknowledges financial support of INFN (iniziative specifiche TEONGRAV and QGSKY). This article is also based upon work from COST action CA15117 (CANTATA), supported by COST (European Cooperation in Science and Technology).

\section{A The action components (2.3) in terms of couplings of parallel and anti-parallel spins}

In this Appendix we use the definitions (2.5) in order to calculate the different terms of (2.3) in terms of couplings of parallel and anti-parallel spins [18]. In particular, we obtain:

$$
\begin{aligned}
\left\langle F^{a b c}, F_{a b c}\right\rangle_{\text {Free-Free }}= & A^{a b} i \sigma_{i j}^{2} \partial_{a}^{i} \psi_{b}^{j}+\sigma_{i j}^{0} \psi^{\dagger a, i} \psi_{a}^{j}-\sigma_{i j}^{1} \psi^{\dagger a, i} \psi_{a}^{j} \\
& +\sigma_{i^{\prime} i}^{0}\left(\psi^{\dagger a, i^{\prime}} i \sigma_{i^{\prime} j}^{0} \sigma_{j k}^{1} \partial^{a, j} \psi_{a}^{k}\right)\left(\psi_{a}^{\dagger i} i \sigma_{i j}^{0} \sigma_{j k}^{1} \partial^{a, j} \psi_{a}^{k}\right) \\
& +\sigma_{i^{\prime} i}^{1}\left(\psi^{\dagger a, i^{\prime}} i \sigma_{i^{\prime} j}^{0} \sigma_{j k}^{1} \partial^{a, j} \psi_{a}^{k}\right)\left(\psi_{a}^{\dagger i} i \sigma_{i j}^{0} \sigma_{j k}^{1} \partial^{a, j} \psi_{a}^{k}\right) \\
& -\sigma_{i^{\prime} i}^{0}\left(\psi^{\dagger a, i^{\prime}} i \sigma_{i^{\prime} j}^{1} \sigma_{j k}^{1} \partial^{a, j} \psi_{a}^{k}\right)\left(\psi_{a}^{\dagger i} i \sigma_{i j}^{1} \sigma_{j k}^{1} \partial^{a, j} \psi_{a}^{k}\right),
\end{aligned}
$$


and

$$
\begin{aligned}
\left\langle\partial^{b} \partial^{a} X^{i}, \partial_{b} \partial_{a} X^{i}\right\rangle= & \varepsilon^{a b c} \varepsilon^{a d e}\left(\partial_{b} \partial_{c} X_{\alpha}^{i}\right)\left(\partial_{e} \partial_{d} X_{\beta}^{i}\right)= \\
& \Psi^{\dagger a, U}\left\langle F_{a b c}, F^{a^{\prime} b c}\right\rangle \Psi_{a^{\prime}}^{L}+\Psi^{\dagger a, L}\left\langle F_{a b c}, F^{a^{\prime} b c}\right\rangle \Psi_{a^{\prime}}^{U}-\Psi^{\dagger a, U}\left\langle F_{a b c}, F^{a^{\prime} b c}\right\rangle \Psi_{a^{\prime}}^{U} \\
& -\Psi^{\dagger a, L}\left\langle F_{a b c}, F^{a^{\prime} b c}\right\rangle \Psi_{a^{\prime}}^{L}+\Psi^{\dagger a, L} \Psi^{\dagger d, U} \partial_{d} \partial^{d^{\prime}}\left\langle F_{a b c}, F^{a^{\prime} b c}\right\rangle \Psi_{a^{\prime}}^{L} \Psi_{d^{\prime}}^{U} \\
& -\Psi^{\dagger a, L} \Psi^{\dagger d, U} \partial_{d}\left\langle F_{a b c}, F^{a^{\prime} b c}\right\rangle \Psi_{a^{\prime}}^{L}-\Psi^{\dagger a, U} \Psi^{\dagger d, L} \partial_{d}\left\langle F_{a b c}, F^{a^{\prime} b c}\right\rangle \Psi_{a^{\prime}}^{U} \\
& +\psi^{\dagger i, U}\left\langle F_{i j k}, F^{i^{\prime} j k}\right\rangle \psi_{i^{\prime}}^{L}+\psi^{\dagger i, L}\left\langle F_{i j k}, F^{i^{\prime} j k}\right\rangle \psi_{i^{\prime}}^{U}-\psi^{\dagger i, U}\left\langle F_{i j k}, F^{i^{\prime} j k}\right\rangle \psi_{i^{\prime}}^{U} \\
& -\psi^{\dagger i, L}\left\langle F_{i j k}, F^{i^{\prime} j k}\right\rangle \psi_{i^{\prime}}^{L}+\psi^{\dagger i, L} \psi^{\dagger m, U} \partial_{m} \partial^{m^{\prime}}\left\langle F_{i j k}, F^{i^{\prime} j k}\right\rangle \psi_{i^{\prime}}^{L} \psi_{m^{\prime}}^{U} \\
& -\psi^{\dagger i, L} \psi^{\dagger m, U} \partial_{m}\left\langle F_{i j k}, F^{i^{\prime} j k}\right\rangle \psi_{i^{\prime}}^{L}-\psi^{\dagger i, U} \psi^{\dagger m, L} \partial_{m}\left\langle F_{i j k}, F^{i^{\prime} j k}\right\rangle \psi_{i^{\prime}}^{U} \\
& +\Psi^{\dagger a, U}\left\langle F_{a b c}, F^{i^{\prime} b c}\right\rangle \psi_{i^{\prime}}^{L}+\Psi^{\dagger a, L}\left\langle F_{a b c}, F^{i^{\prime} b c}\right\rangle \psi_{i^{\prime}}^{U}-\Psi^{\dagger a, U}\left\langle F_{a b c}, F^{i^{\prime} b c}\right\rangle \psi_{i^{\prime}}^{U} \\
& -\Psi^{\dagger a, L}\left\langle F_{a b c}, F^{i^{\prime} b c}\right\rangle \psi_{i^{\prime}}^{L}+\Psi^{\dagger a, L} \Psi^{\dagger d, U} \partial_{d} \partial^{i^{\prime}}\left\langle F_{a b c}, F^{j^{\prime} b c}\right\rangle \psi_{j^{\prime}}^{L} \psi_{i^{\prime}}^{U} \\
& -\Psi^{\dagger a, L} \Psi^{\dagger d, U} \partial_{d}\left\langle F_{a b c}, F^{i^{\prime} b c}\right\rangle \psi_{i^{\prime}}^{L}-\Psi^{\dagger a, U} \Psi^{\dagger d, L} \partial_{d}\left\langle F_{a b c}, F^{i^{\prime} b c}\right\rangle \psi_{i^{\prime}}^{U} .
\end{aligned}
$$

Additionally, since $\left(\psi_{a}^{U}\right)^{2}=0$ and $\left(\psi_{a}^{L}\right)^{2}=0$, we get

$$
\begin{aligned}
F(X)= & \Sigma_{j} X_{j}^{2}=\Sigma_{j}\left[\psi_{a}^{U} A^{a b, j} \psi_{b}^{L}-\psi_{a}^{L} A_{a b, j} \psi_{b}^{U}\right]^{2} \\
= & \Sigma_{j}\left(\psi_{a}^{U}\right)^{2}\left(A^{a b, j}\right)^{2}\left(\psi_{b}^{L}\right)^{2}+\left(\psi_{a}^{L}\right)^{2}\left(A_{a b, j}\right)^{2}\left(\psi_{b}^{U}\right)^{2} \\
& -\left(\psi_{a}^{U} A^{a b, j} \psi_{b}^{L}\right)\left(\psi_{a}^{L} A_{a b, j} \psi_{b}^{U}\right)-\left(\psi_{a}^{L} A_{a b, j} \psi_{b}^{U}\right)\left(\psi_{a}^{U} A^{a b, j} \psi_{b}^{L}\right)=0
\end{aligned}
$$

and

$$
\left\langle\left[X^{k}, X^{i}, X^{j}\right],\left[X_{k}, X_{i}, X_{j}\right]\right\rangle=\Sigma_{n} \Sigma_{m} \alpha_{n+m}\left(\psi^{L}\right)^{2 n}\left(\psi^{U}\right)^{2 m}=0 .
$$

In the above expressions $a, b, c$ are indices of bound electrons, while $i, j, k$ are indices of free electrons, and $U, L$ refers to upper and lower spins. Additionally, we have used the Pauli matrices definition as: $\sigma_{i j}^{1}=\left(\begin{array}{ll}0 & 1 \\ 1 & 0\end{array}\right), \sigma_{i j}^{0}=\left(\begin{array}{ll}1 & 0 \\ 0 & 1\end{array}\right), \sigma_{i j}^{2}=\left(\begin{array}{cc}-i \\ i & 0\end{array}\right)$. 


\section{B Expression of $\left\langle\partial^{b} \partial^{a} X^{i}, \partial_{b} \partial_{a} X^{i}\right\rangle$ in terms of curvatures}

The term $\left\langle\partial^{b} \partial^{a} X^{i}, \partial_{b} \partial_{a} X^{i}\right\rangle$ is expressed in terms of curvatures as:

$$
\begin{aligned}
& \left\langle\partial^{b} \partial^{a} X^{i}, \partial_{b} \partial_{a} X^{i}\right\rangle=\Psi^{\dagger a, U} R_{a a^{\prime}}^{a n t i-p a r a l l e l} \Psi^{a^{\prime}, L}+\Psi^{\dagger a, L} R_{a a^{\prime}}^{\text {anti-parallel }} \Psi^{a^{\prime}, U} \\
& -\Psi^{\dagger a, U} R_{a a^{\prime}}^{\text {parallel }} \Psi^{a^{\prime}, U}-\Psi^{\dagger a, L} R_{a a^{\prime}}^{\text {parallel }} \Psi^{a^{\prime}, L} \\
& +\Psi^{\dagger a, L} \Psi^{\dagger d, U} \partial_{d} \partial^{d^{\prime}}\left(R_{a a^{\prime}}^{\text {parallel }}+R_{a a^{\prime}}^{\text {anti-parallel }}\right) \Psi^{a^{\prime}, L} \Psi_{d^{\prime}}^{U} \\
& -\Psi^{\dagger a, L} \Psi^{\dagger d, U} \partial_{d}\left(R_{a a^{\prime}}^{\text {parallel }}+R_{a a^{\prime}}^{\text {anti-parallel }}\right) \Psi^{a^{\prime}, L} \\
& -\Psi^{\dagger a, U} \Psi^{\dagger d, L} \partial_{d}\left(R_{a a^{\prime}}^{\text {parallel }}+R_{a a^{\prime}}^{\text {anti-parallel }}\right) \Psi^{a^{\prime}, U} \\
& +\psi^{\dagger i, U} R_{i i^{\prime}}^{\text {anti-parallel }} \psi^{i^{\prime}, L}+\psi^{\dagger i, L} R_{i i^{\prime}}^{\text {anti-parallel }} \psi^{i^{\prime}, U} \\
& -\psi^{\dagger i, U} R_{i i^{\prime}}^{\text {parallel }} \psi^{i^{\prime}, U}-\psi^{\dagger i, L} R_{i i^{\prime}}^{\text {parallel }} \psi^{i^{\prime}, L} \\
& +\psi^{\dagger i, L} \psi^{\dagger m, U} \partial_{m} \partial^{m^{\prime}}\left(R_{i j^{\prime}}^{\text {parallel }}+R_{i j^{\prime}}^{\text {anti-parallel }}\right) \psi^{i^{\prime}, L} \psi_{m^{\prime}}^{U} \\
& -\psi^{\dagger i, L} \psi^{\dagger m, U} \partial_{m}\left(R_{i j^{\prime}}^{\text {parallel }}+R_{i j^{\prime}}^{\text {anti-parallel }}\right) \psi^{i^{\prime}, L} \\
& -\psi^{\dagger i, U} \psi^{\dagger m, L} \partial_{m}\left(R_{i j^{\prime}}^{\text {parallel }}+R_{i j^{\prime}}^{\text {anti-parallel }}\right) \psi^{i^{\prime}, U} \\
& +\Psi^{\dagger a, U} R_{a i^{\prime}}^{a n t i-\text { parallel }} \psi^{i^{\prime}, L}+\Psi^{\dagger a, L} R_{a i^{\prime}}^{a n t i-\text { parallel }} \psi^{i^{\prime}, U} \\
& -\Psi^{\dagger a, U} R_{a i^{\prime}}^{\text {parallel }} \psi^{i^{\prime}, U}-\Psi^{\dagger a, L} R_{a i^{\prime}}^{\text {parallel }} \psi^{i^{\prime}, L} \\
& +\Psi^{\dagger a, L} \Psi^{\dagger d, U} \partial_{d} \partial^{i^{\prime}}\left(R_{a i^{\prime}}^{\text {parallel }}+R_{a i^{\prime}}^{\text {anti-parallel }}\right) \psi_{j^{\prime}}^{L} \psi^{i^{\prime}, U} \\
& -\Psi^{\dagger a, L} \Psi^{\dagger d, U} \partial_{d}\left(R_{a i^{\prime}}^{\text {parallel }}+R_{a i^{\prime}}^{\text {anti-parallel }}\right) \psi^{i^{\prime}, L} \\
& -\Psi^{\dagger a, U} \Psi^{\dagger d, L} \partial_{d}\left(R_{a i^{\prime}}^{\text {parallel }}+R_{a i^{\prime}}^{\text {anti-parallel }}\right) \psi^{i^{\prime}, U} \\
& \approx\left(R_{\text {Free-Free }}^{\text {parallel }}\right)^{2}+\left(R_{\text {Free-Free }}^{\text {anti-parallel }}\right)^{2}+\left(R_{\text {Free-Bound }}^{\text {parallel }}\right)^{2} \\
& +\left(R_{\text {Free-Bound }}^{\text {anti-parallel }}\right)^{2}+\left(R_{\text {Bound-Bound }}^{\text {parallel }}\right)^{2}+\left(R_{\text {Bound-Bound }}^{\text {anti-parallel }}\right)^{2} \\
& +\left(R_{\text {Free-Free }}^{\text {paralle }} R_{\text {Free-Free }}^{\text {anti-parallel }}\right) \partial^{2}\left(R_{\text {Free-Free }}^{\text {parallel }}+R_{\text {Free-Free }}^{\text {anti-parallel }}\right) \\
& +\left(R_{\text {Free-Bound }}^{\text {parallel }} R_{\text {Free-Bound }}^{\text {anti-parallel }}\right) \partial^{2}\left(R_{\text {Free-Bound }}^{\text {parallel }}+R_{\text {Free-Bound }}^{\text {anti-parallel }}\right) \\
& +\left(R_{\text {Bound-Bound }}^{\text {parallel }} R_{\text {Bound-Bound }}^{\text {anti-parallel }}\right) \partial^{2}\left(R_{\text {Bound-Bound }}^{\text {parallel }}+R_{\text {Bound-Bound }}^{\text {anti-parallel }}\right),
\end{aligned}
$$

where $R_{\text {Bound-Bound }}^{\text {anti-parallel }}$ is the curvature produced by the interaction of two bound anti-parallel electrons, $R_{\text {Bound-Bound }}^{\text {parallel }}$ is the curvature created by interaction of two bound parallel electrons, $R_{\text {Free-Free }}^{\text {anti-parallel }}$ is the curvature produced by the interaction of two free anti-parallel electrons, $R_{\text {Free-Free }}^{\text {parallel }}$ is the curvature created by interaction of two free parallel electrons, $R_{\text {Free-Bound }}^{\text {anti-parallel }}$ is the curvature produced by the interaction of free and bound anti-parallel electrons and $R_{\text {Free-Free }}^{\text {parallel }}$ is the curvature created by interaction of free and bound parallel electrons.

\section{Defects and $f(R)$ gravity}

We can show that each defect produces a particular form of extended gravity. For example, in a graphene without defect where curvature is generated by parallel spins and neutralized by curvatures of anti-parallel spins, we have the following form of $f(R)$ function: 


$$
\begin{aligned}
f(R)=\left\{2\left(1-m_{g}^{2}\right)\right. & {\left[\left(R_{\text {Free-Free }}^{\text {parallel }}\right)^{2}+\left(R_{\text {Free-Bound }}^{\text {parallel }}\right)^{2}+\left(R_{\text {Bound }- \text { Bound }}^{\text {parallel }}\right)^{2}\right.} \\
& +\left(R_{\text {Free }- \text { Free }}^{\text {parallel }} R_{\text {Free-Free }}^{\text {anti-parallel }}\right) \partial^{2}\left(R_{\text {Free-Free }}^{\text {parallel }}\right) \\
& +\left(R_{\text {Free-Bound }}^{\text {parallel }} R_{\text {Free-Bound }}^{\text {anti-Parallel }}\right) \partial^{2}\left(R_{\text {Free-Bound }}^{\text {parallel }}\right) \\
& \left.\left.+\left(R_{\text {Bound-Bound }}^{\text {parallel }} R_{\text {Bound-Bound }}^{\text {anti-pallel }}\right) \partial^{2}\left(R_{\text {Bound-Bound }}^{\text {parallel }}\right)\right]\right\}^{N},
\end{aligned}
$$

where $\mathrm{N}$ is the number of atoms in graphene.

For a graphene structure with heptagonal defects where (i.e. the curvature produced by parallel spins is larger than the curvature produced by anti-parallel spins), some extra terms will be added and the form of $f(R)$ gravity is:

$$
\begin{aligned}
& f(R)=\left\{-\left(1-m_{g}^{2}\right)\left[\left(R_{\text {Free }- \text { Free }}^{\text {parallel }}\right)^{2}+\left(R_{\text {Free-Free }}^{\text {anti-parallel }}\right)^{2}+\left(R_{\text {Free-Bound }}^{\text {parallel }}\right)^{2}\right.\right. \\
& +\left(R_{\text {Free-Bound }}^{\text {anti-parallel }}\right)^{2}+\left(R_{\text {Bound-Bound }}^{\text {parallel }}\right)^{2}+\left(R_{\text {Bound-Bound }}^{\text {anti-parallel }}\right)^{2} \\
& +\left(R_{\text {Free-Free }}^{\text {parallel }} R_{\text {Free-Free }}^{\text {anti-parallel }}\right) \partial^{2}\left(R_{\text {Free-Free }}^{\text {parallel }}+R_{\text {Free-Free }}^{\text {anti-parallel }}\right) \\
& +\left(R_{\text {Free-Bound }}^{\text {parallel }} R_{\text {Free-Bound }}^{\text {anti-parallel }}\right) \partial^{2}\left(R_{\text {Free-Bound }}^{\text {parallel }}+R_{\text {Free-Bound }}^{\text {anti-parallel }}\right) \\
& \left.+\left(R_{\text {Bound-Bound }}^{\text {parallel }} R_{\text {Bound-Bound }}^{\text {anti-parallel }}\right) \partial^{2}\left(R_{\text {Bound-Bound }}^{\text {parallel }}+R_{\text {Bound-Bound }}^{\text {anti-parallel }}\right)\right] \\
& -m_{g}^{2} \lambda^{2} \delta_{\rho_{1} \sigma_{1}}^{\mu_{1} \nu_{1}}\left[R_{\text {Free-Free }, \mu_{1} \nu_{1}}^{\text {parallel, } \rho_{1} \sigma_{1}}+R_{\text {Bound }- \text { Bound, } \mu_{1} \nu_{1}}^{\text {parallel }, \rho_{1} \sigma_{1}}+R_{\text {Free-Bound, } \mu_{1} \nu_{1}}^{\text {parallel }, \rho_{1} \sigma_{1}}\right. \\
& \left.\left.-R_{\text {Free-Free, } \mu_{1} \nu_{1}}^{\text {anti-parallel, } \rho_{1} \sigma_{1}}-R_{\text {Bound-Bound, } \mu_{1} \nu_{1}}^{\text {anti-parallel, } \rho_{1} \sigma_{1}}-R_{\text {Free-Bound, } \mu_{1} \nu_{1}}^{\text {anti-parallel, } \sigma_{1} \sigma_{1}}\right]\right\}^{N},
\end{aligned}
$$

where $\mathrm{N}$ is the number of heptagonal molecules.

For a graphene structure with pentagonal defects (i.e. the curvature produced by antiparallel spins is larger than the curvature produced by parallel spins), the sign of $m_{g}^{2} \lambda^{2}$ changes:

$$
\begin{aligned}
& f(R)=\left\{-\left(1-m_{g}^{2}\right)\left[\left(R_{\text {Free-Free }}^{\text {parallel }}\right)^{2}+\left(R_{\text {Free-Free }}^{\text {anti-parallel }}\right)^{2}+\left(R_{\text {Free-Bound }}^{\text {parallel }}\right)^{2}\right.\right. \\
& +\left(R_{\text {Free-Bound }}^{\text {anti-parallel }}\right)^{2}+\left(R_{\text {Bound-Bound }}^{\text {parallel }}\right)^{2}+\left(R_{\text {Bound-Bound }}^{\text {anti-parallel }}\right)^{2} \\
& +\left(R_{\text {Free-Free }}^{\text {parallel }} R_{\text {Free-Free }}^{\text {anti-parallel }}\right) \partial^{2}\left(R_{\text {Free-Free }}^{\text {parallel }}+R_{\text {Free-Free }}^{\text {anti-parallel }}\right) \\
& +\left(R_{\text {Free-Bound }}^{\text {parallel }} R_{\text {Free-Bound }}^{\text {anti-parallel }}\right) \partial^{2}\left(R_{\text {Free-Bound }}^{\text {parallel }}+R_{\text {Free-Bound }}^{\text {anti-parallel }}\right) \\
& \left.+\left(R_{\text {Bound-Bound }}^{\text {parallel }} R_{\text {Bound-Bound }}^{\text {anti-parallel }}\right) \partial^{2}\left(R_{\text {Bound-Bound }}^{\text {parallel }}+R_{\text {Bound-Bound }}^{\text {anti-parallel }}\right)\right] \\
& +m_{g}^{2} \lambda^{2} \delta_{\rho_{1} \sigma_{1}}^{\mu_{1} \nu_{1}}\left[R_{\text {Free-Free }, \mu_{1} \nu_{1}}^{\text {parallel, } \rho_{1} \sigma_{1}}+R_{\text {Bound }- \text { Bound }, \mu_{1} \nu_{1}}^{\text {parallel }, \rho_{1} \sigma_{1}}+R_{\text {Free-Bound }, \mu_{1} \nu_{1}}^{\text {parallel, } \rho_{1} \sigma_{1}}\right. \\
& \left.\left.-R_{\text {Free-Free }, \mu_{1} \nu_{1}}^{\text {anti-parallel } \rho_{1} \sigma_{1}}-R_{\text {Bound-Bound, } \mu_{1} \nu_{1}}^{\text {anti-parallel }, \rho_{1} \sigma_{1}}-R_{\text {Free-Bound, } \mu_{1} \nu_{1}}^{\text {anti-parallel }, \sigma_{1}}\right]\right\}^{N},
\end{aligned}
$$


Here, $\mathrm{N}$ is the number of pentagonal molecules. Thus, the gravity which is produced by hexagonal molecules is very different from gravity produced by pentagonal or heptagonal molecules. The sign of parameter $m_{g}^{2} \lambda^{2}$ plays a special role in this modelling.

\section{References}

[1] S. Bera, A. Arnold, F. Evers, R. Narayanan, P. Woelfle, Elastic properties of graphene flakes: boundary effects and lattice vibrations Phys. Rev. B 82, 195445 (2010), [arXiv:1003.4429].

[2] V. Hfner, J. Schindler, N. Weik, T. Mayer, S. Balakrishnan, R. Narayanan, S. Bera and F. Evers, Density of states in graphene with vacancies: midgap power law and frozen multifractality, Phys. Rev. Lett. 113, no. 18, 186802 (2014), [arXiv:1404.6138].

[3] P. M. Ostrovsky, I. V. Protopopov, E. J. Knig, I. V. Gornyi, A. D. Mirlin, M. A. Skvortsov, Density of states in a two-dimensional chiral metal with vacancies, Phys. Rev. Lett. 113, 186803 (2014), [arXiv: 1404.6139].

[4] M. V. Ulybyshev and M. I. Katsnelson, Magnetism and interaction-induced gap opening in graphene with vacancies or hydrogen adatoms: Quantum Monte Carlo study, Phys. Rev. Lett. 114, no. 24, 246801 (2015), [arXiv: 1502.01184].

[5] A. A. Stabile, A. Ferreira, J. Li, N. M. R. Peres, J. Zhu, Electrically tunable resonant scattering in fluorinated bilayer graphene, Phys. Rev. B 92, 121411(R) (2015), [arXiv: 1510.01573].

[6] A. Dasgupta, S. Bera, F. Evers, M. J. van Setten, Quantum Size Effects in the Atomistic Structure of Armchair-Nanoribbons, Phys. Rev. B 85, 125433 (2012), [arXiv:1111.3593].

[7] J. Wilhelm, M. Walz, F. Evers, Ab initio quantum transport through armchair graphene nanoribbons: Streamlines in the current density, Phys. Rev. B 89, 195406 (2014), [arXiv: 1405. 3205].

[8] E. A. Kochetov, V. A. Osipov and R. Pincak, Electronic properties of disclinated flexible membrane beyond the inextensional limit: Application to graphene, J. Phys. Condens. Matter 22, 395502 (2010), [arXiv: 1005.3232].

[9] J. Wilhelm, M. Walz, F. Evers, Ab initio spin-flip conductance of hydrogenated graphene nanoribbons: Spin-orbit interaction and scattering with local impurity spins, Phys. Rev. B 92, 014405 (2015), [arXiv: 1504.06720].

[10] Gonzalo J. Olmo, D. Rubiera-Garcia, Palatini $f(R)$ black holes in nonlinear electrodynamics, Phys. Rev. D 84, 124059 (2011), [arXiv:1110.0850].

[11] Cosimo Bambi, Alejandro Cardenas-Avendano, Gonzalo J. Olmo, D. Rubiera-Garcia, Wormholes and nonsingular space-times in Palatini $f(R)$ gravity, Phys. Rev. D 93, 064016 (2016), [arXiv: 1511.03755].

[12] Francisco S. N. Lobo, Gonzalo J. Olmo, D. Rubiera-Garcia, Crystal clear lessons on the microstructure of space-time and modified gravity, Phys. Rev. D 91, 124001 (2015), [arXiv: 1412 .4499].

[13] M. A. H. Vozmediano, M. I. Katsnelson, F. Guinea, Gauge fields in graphene, Phys. Rept. 496, 109 (2010), [arXiv: 1003.51790].

[14] A. Sepehri, Cosmology from quantum potential in braneanti-brane system, Phys. Lett. B 748, 328 (2015), [arXiv: 1508.01407]. 
[15] A. Sepehri, F. Rahaman, S. Capozziello, A. F. Ali and A. Pradhan, Emergence and oscillation of cosmic space by joining M1-branes, Eur. Phys. J. C 76, no. 5, 231 (2016), [arXiv: 1604.02451].

[16] A. Sepehri, F. Rahaman, A. Pradhan and I. H. Sardar, Emergence and Expansion of Cosmic Space in BIonic system, Phys. Lett. B 741, 92 (2015), [arXiv: 1501.00428].

[17] A. Sepehri, F. Rahaman, M. R. Setare, A. Pradhan, S. Capozziello and I. H. Sardar, Unifying inflation with late-time acceleration by a BIonic system, Phys. Lett. B 747, 1 (2015), [arXiv: 1505.05105].

[18] A. Sepehri, BornInfeld extension of Lovelock brane gravity in the system of M0-branes and its application for the emergence of Pauli exclusion principle in BIonic superconductors, Phys. Lett. A 380, 2247 (2016).

[19] A. Sepehri, M. R. Setare and S. Capozziello, Emergence and expansion of cosmic space as due to M0-branes, Eur. Phys. J. C 75, no. 12, 618 (2015), [arXiv:1512.04840].

[20] J.A.R. Cembranos, A. Dobado, A.L. Maroto, Brane-world dark matter, Phys. Rev. Lett. 90, 241301 (2003), [arXiv:0302041].

[21] Jose A. R. Cembranos, Antonio L. Maroto, Disformal scalars as dark matter candidates: Branon phenomenology, International Journal of Modern Physics A 31, 1630015 (2016), [arXiv: 1602.07270].

[22] J.A.R. Cembranos, A. de la Cruz-Dombriz, V. Gammaldi, A.L. Maroto, Detection of branon dark matter with gamma ray telescopes, Phys. Rev. D. 85, 043505 (2012), [arXiv: 1111.4448].

[23] L. Randall and R. Sundrum, A Large Mass Hierarchy from a Small Extra Dimension, Phys. Rev. Lett. 83, 3370 (1999), [arXiv: 9905221].

[24] Gonzalo J. Olmo, D. Rubiera-Garcia, Brane-world and loop cosmology from a gravity-matter coupling perspective, Phys. Lett. B. 740, 73 (2015), [arXiv:1405.7184].

[25] M.D. Maia, Edmundo M. Monte, J.M.F. Maia, The accelerating universe in brane-world cosmology, Phys. Lett. B. 585, 11 (2004), [arXiv: 0208223].

[26] S. Capozziello and M. De Laurentis, Extended Theories of Gravity, Phys. Rept. 509, 167 (2011), [arXiv: 1108.6266].

[27] S. Capozziello and M. Francaviglia, Extended Theories of Gravity and their Cosmological and Astrophysical Applications, Gen. Rel. Grav. 40 (2008) 357 [arXiv:0706.1146 [astro-ph]] .

[28] J. Bagger and N. Lambert, Gauge symmetry and supersymmetry of multiple M2-branes, Phys. Rev. D 77, 065008 (2008), [arXiv:0711.0955].

[29] R. C. Myers, Dielectric branes, JHEP 9912, 022 (1999), [arXiv:hep-th/9910053].

[30] A. Gustavsson, Algebraic structures on parallel M2-branes, Nucl. Phys. B 811, 66 (2009), [arXiv:0709.1260].

[31] N. R. Constable, R. C. Myers and O. Tafjord, NonAbelian brane intersections, JHEP 0106, 023 (2001), [arXiv:hep-th/0102080].

[32] A. Sepehri, R. Pincak and A. F. Ali, Emergence of $F(R)$ gravity-analogue due to defects in graphene, [arXiv:1606.02039].

[33] P. M. Ho and Y. Matsuo, M5 from M2, JHEP 0806, 105 (2008), [arXiv:0804.3629]. 
[34] S. Mukhi and C. Papageorgakis, M2 to D2, JHEP 0805, 085 (2008), [arXiv:0803.3218].

[35] P. D. Mannheim and J. J. Poveromo, Gravitational analog of Faraday's law via torsion and a metric with an antisymmetric part, Gen. Rel. Grav. 46, no. 10, 1795 (2014), [arXiv: 1406.1470].

[36] W. T. Ni, Spacetime structure and asymmetric metric from the premetric formulation of electromagnetism, Phys. Lett. A 379, 1297 (2015), [arXiv:1411.0460].

[37] R. Pincak, J. Smotlacha, Analogies in electronic properties of graphene wormhole and perturbed nanocylinder, Eur. Phys. J. B 86, 480 (2013).

[38] J. Gonzalez and J. Herrero, Graphene wormholes: A Condensed matter illustration of Dirac fermions in curved space, Nucl. Phys. B 825, 426 (2010), [arXiv:0909.3057].

[39] V. Atanasov, A. Saxena, Superconducting tunneling spectroscopy of graphene and graphene nanostructures, J.Phys: Cond. Mat. 23 (2011) 175301, [arXiv:1101.5243].

[40] G. Ozin, A.C. Arsenault, L. Cademartiri, Nanochemistry: A Chemical Approach to Nanomateria ls 2nd Eds. (Royal Society of Chemistry, 2008) 820p. ISBN 978-1847558954.

[41] K. J. Klabunde, M. Richards, eds. (2009). Nanoscale Materials in Chemistry (2nd ed.). Wiley. ISBN 978-0-470-22270-6.

[42] M. Visser, C. Barceló, S. Liberati, Analogue models of and for gravity Gen. Rel. Grav. 34 $1719(2002)$. 